\title{
Upregulated miR-203a-3p and its potential molecular mechanism in breast cancer: A study based on bioinformatics analyses and a comprehensive meta-analysis
}

\author{
KAI-TENG CAI, CAI-XIA FENG, JIN-CHE ZHAO, RONG-QUAN HE, JIE MA and JIN-CAI ZHONG \\ Department of Medical Oncology, First Affiliated Hospital of Guangxi Medical University, \\ Nanning, Guangxi Zhuang Autonomous Region 530021, P.R. China
}

Received November 4, 2017; Accepted August 31, 2018

DOI: $10.3892 / \mathrm{mmr} .2018 .9543$

\begin{abstract}
Breast cancer (BC) has been identified as the leading malignancy in women worldwide. However, the potential molecular mechanism of microRNA (miR)-203a-3p in BC remains to be elucidated. The present study evaluated the expression of miR-203a-3p in BC and adjacent normal tissue in several publically available datasets. The distinguishability of precursor miR-203a and miR-203a-3p in BC tissue and adjacent breast tissue was assessed using receiver operating characteristic (ROC) and summarized ROC (sROC) approaches. In addition, gene ontology (GO) enrichment, Kyoto Encyclopedia of Genes and Genomes pathway analysis and protein-protein interaction analysis were performed to determine the potential molecular mechanism of miR-203a-3p in BC. It was identified that the expression of precursor miR-203a was markedly upregulated in 1,077 BC tissue samples compared to 104 adjacent breast tissue samples from The Cancer Genome Atlas. Additionally, an increasing trend in miR-203a-3p expression was observed in 756 BC tissue samples compared with 76 adjacent breast tissue samples from the University of California Santa Cruz Xena project. In addition, a comprehensive meta-analysis suggested that the expression of miR-203a-3p was markedly increased in 2,444 BC tissue samples compared with 559 adjacent breast tissue samples. The area under the curve of the ROC and sROC revealed that miR-203a-3p expression was able to distinguish between $\mathrm{BC}$ tissue and adjacent breast tissue. However, miR-203a-3p exhibited no prognostic value in $\mathrm{BC}$. The results of GO enrichment demonstrated that the miR-203a target genes were associated with 'plasma membrane integrity', 'cell surface receptor linked signal and transduction' and
\end{abstract}

Correspondence to: Dr Jin-Cai Zhong, Department of Medical Oncology, First Affiliated Hospital of Guangxi Medical University, 6 Shuangyong Road, Nanning, Guangxi Zhuang Autonomous Region 530021, P.R. China

E-mail: zhongjincai_gxmu@163.com

Key words: microRNA-203a-3p, breast cancer, the cancer genome atlas, gene expression omnibus, target gene '3',5'-cyclic nucleotide phosphodiesterase activity'. 'Purine metabolism' was identified as the pathway with the most enrichment of miR-203a-3p target genes in BC. The present study also identified insulin-like growth factor receptor (IGF1) as a hub gene associated with miR-203a in BC. In summary, miR-203a-3p may enhance the development and oncogenesis of $\mathrm{BC}$, and IGF1 was defined as a hub gene of miR-203a-3p in BC.

\section{Introduction}

Breast cancer (BC) ranks as the most common malignancy in women worldwide and ranks as the second most common cause of cancer-associated mortality $(1,2)$. The incidence of $\mathrm{BC}$ is increasing; the latest cancer statistics from the USA estimated that the expected numbers of new cancer cases and mortalities could reach 66,120 and 40,920, respectively, in 2018 (3). The human epidermal growth factor receptor 2 (HER2), progesterone receptor (PR), and estrogen receptor (ER) were established as the biomarkers of $\mathrm{BC}$, and $\mathrm{BC}$ can be classified into four molecular subtypes depending on the expression of HER2, PR and ER: HER2(+), triple negative breast cancer, Luminal A and Luminal B. Currently, advanced therapeutic approaches have been applied in BC cases to improve the 5-year survival rate based on the above classification, including chemotherapy, surgical techniques and adjuvant radiotherapy (4-8). Nevertheless, the 5-year survival rate of $\mathrm{BC}$ patients with distant metastasis and tumor progression is only $26 \%$. Additionally, only $1.9 \%$ of patients under 50 with $\mathrm{BC}$ received a $\mathrm{BC}$ diagnosis, but $\sim 80 \%$ of $\mathrm{BC}$ patients over 50 received a $\mathrm{BC}$ diagnosis. Therefore, an improved understanding of potential treatment targets is imperative to improve the 5-year survival rate and diagnosis of patients with $\mathrm{BC}(9,10)$.

MicroRNAs (miRNAs/miRs) are small, non-coding RNAs of $\sim 22$ nucleotides. They regulate the expression of proteins by silencing the transcripts of target genes or inhibiting the translation of mRNA $(11,12)$. Extensive studies have established that miRNAs are crucial in the diagnosis, proliferation, prognosis, invasion, apoptosis, migration and metastasis of cancer (13-17). For example, Liang et al suggested that miRNA-10b was a suppressor in BC growth, migration, proliferation and invasion (18). Du et al reported that miR-124 
inhibited the proliferation and migration of $\mathrm{BC}$ by targeting snail family transcriptional repressor 2 (19).

Located at 14q32.33 chromosome, miR-203a-3p may possess a vital role in cancer. It has been reported that miR-203a-3p can suppress hepatocellular carcinoma progression by targeting homeobox D3 through the EGFR signaling pathway (20). However, only one study has examined the role of miR-203a-3p in BC based on 109 BC cases and matched normal breast (21). Therefore, it is critical to establish the molecular mechanism of miR-203a-3p in BC with a large number of samples. The present study estimated the expression of precursor miR-203a and miR-203a-3p in BC tissue and adjacent breast tissue by combing data from The Cancer Genome Atlas (TCGA), Gene Expression Omnibus (GEO) and University of California Santa Cruz (UCSC) Xena projects. In addition, the potential molecular mechanisms of miR-203a-3p in $\mathrm{BC}$ were investigated through gene ontology (GO) enrichment, Kyoto Encyclopedia of Genes and Genomes (KEGG) pathways analysis and protein-protein interaction (PPI).

\section{Materials and methods}

Expression of miRNA in TCGA and UCSC Xena projects. The TCGA data with level 3 miRNA-Seq profiles and full annotation of clinical parameters were acquired from TCGA (http://cancergenome.nih.gov/) (22). Additionally, the expression of miR-203a-3p was downloaded from the UCSC Xena project (http://xena.ucsc.edu/) (23).

Selection of BC microarrays from GEO data. The GEO (https://www.ncbi.nlm.nih.gov/geo/) (24) was used to download BC-associated microarrays with the following prerequisites: (Breast OR mammary) AND (carcinoma OR tumor OR tumor OR neoplas* OR adenocarcinoma OR malignan* OR cancer). Microarrays were selected using the following criteria: The microarrays should include $\mathrm{BC}$ tissue and adjacent breast tissue, and the expression of miR-203a-3p in the two types of tissue should be provided. A gene expression profile named GSE50697 was screened to identify the differentially expressed genes (DEGs).

Selectionofprospective DEGsandtargetgenes ofmiR-203a-3p in $B C$. The prospective target genes of miR-203a-3p were obtained from miRWalk2.0 databases (http://zmf. umm.uni-heidelberg.de/apps/zmf/mirwalk2/) (25), which included 12 online prediction tools: miRDB, miRNAMap, RNAhybrid, miRBridge, miRMap, PICTAR2, PITA, MicroT4, TargetScan, miRWalk2.0, miRanda and RNA22. Prospective target genes were selected if they appeared at least four times in the above 12 online prediction tools to augment the accuracy of the prediction. Gene Expression Profiling Interactive Analysis (http://gepia.cancer-pku. cn/index.html) (26) was performed to acquire the DEGs from TCGA with $\mathrm{P}<0.05$ and $\log 2$ fold change $>1$. DEGs from GEO were achieved by using GEO2R (ncbi.nlm.nih. gov/geo/geo2r/) to analyze GSE50697 with $\mathrm{P}<0.05$ and $\log _{2}$ fold change $<-1$.

Bioinformatics analyses. Venn diagrams were created to obtain the intersection of prospective target genes, such as DEGs from GEO and DEGs from TCGA, and to identify the potential target genes of miR-203a-3p in BC (27). Subsequently, GO and KEGG pathway analyses were used to confirm the potential mechanism of miR-203a-3p in BC $(28,29)$. PPI analysis was also undertaken using the Search Tool for the Retrieval of Interacting Genes/Proteins (STRING) version 9.1 database (https://string-db.org/) (30-32) to generate an association between the possible target genes and hub genes were selected by counting the number of edges and nodes.

Statistical analyses. Student's t-test was used to evaluate statistically significant differences between two groups. Simultaneously, one way analysis variance and Dunnett's test were carried out to estimate statistically significant differences between multiple groups. The receiver operating characteristic (ROC) curve was adopted to assess the distinguishability of precursor miR-203a and miR-203a-3p between $\mathrm{BC}$ tissue and adjacent breast tissue. The Kaplan-Meier survival analysis was undertaken to evaluate the prognostic value of precursor miR-203a in BC. The log-rank test was used to compare high and low precursor miR-203a expression groups. STATA version 12.0 (StataCorp LP, College Station, TX, USA) was used to perform the statistical analyses of the meta-analysis in the present study. The standard mean difference (SMD) with a random effects model was used to measure the expression of miR-203a-3p in BC tissue and adjacent breast tissue. To identify the heterogeneity of the studies, a heterogeneity test was performed and the level of $\mathrm{I}^{2}$ calculated simultaneously. An influence analysis was also conducted to ensure the source of heterogeneity. Concurrently, a funnel plot asymmetry test was undertaken to assess the publication bias, with $\mathrm{P}<0.05$ indicating significant publication bias. The distinguishability of miR-203a-3p in $\mathrm{BC}$ tissue and adjacent breast tissue was estimated using a summarized ROC (sROC) approach, with an area under the curve $(A U C)>0.7$ indicting an ability to distinguish miR-203a-3p in BC. Spearman's correlation analysis was used to verify the correlation between miR-203a-3p and target genes based on TCGA data. $r>0$ and $r<0$ indicated a positive and negative correlation, respectively,

\section{Results}

Clinical value of precursor miR-203a and miR-203a-3p in $B C$, using TCGA and UCSC Xena data. The expression of precursor miR-203a was markedly elevated in 1,077 BC tissue cases compared to 104 adjacent breast tissue cases according to TCGA project data $(13.45 \pm 1.97$ vs. $11.69 \pm 1.72, \mathrm{P}<0.001$; Fig. 1A). Subsequently, the expression of miR-203a-3p was substantially upregulated in $756 \mathrm{BC}$ tissue cases compared to 76 adjacent breast tissue cases in UCSC Xena project data $(11.68 \pm 1.97$ vs. $10.49 \pm 1.05$; $\mathrm{P}<0.001$; Fig. 1B). Regarding the distinguishability of precursor miR-203a and miR-203a-3p, the AUC of ROC curve was 0.775 ( $\mathrm{P}<0.0001$; Fig. 1C) with a sensitivity of $59.24 \%$ and a specificity of $89.42 \%$, which implied that precursor miR-203a could be used to distinguish between BC tissue and adjacent breast tissue. The AUC of ROC in the UCSC Xena project was $0.756(\mathrm{P}<0.0001$; Fig. 1D) with a sensitivity of $61.51 \%$ and a specificity of $88.16 \%$, which indicated that miR-203a-3p could be used to 
A

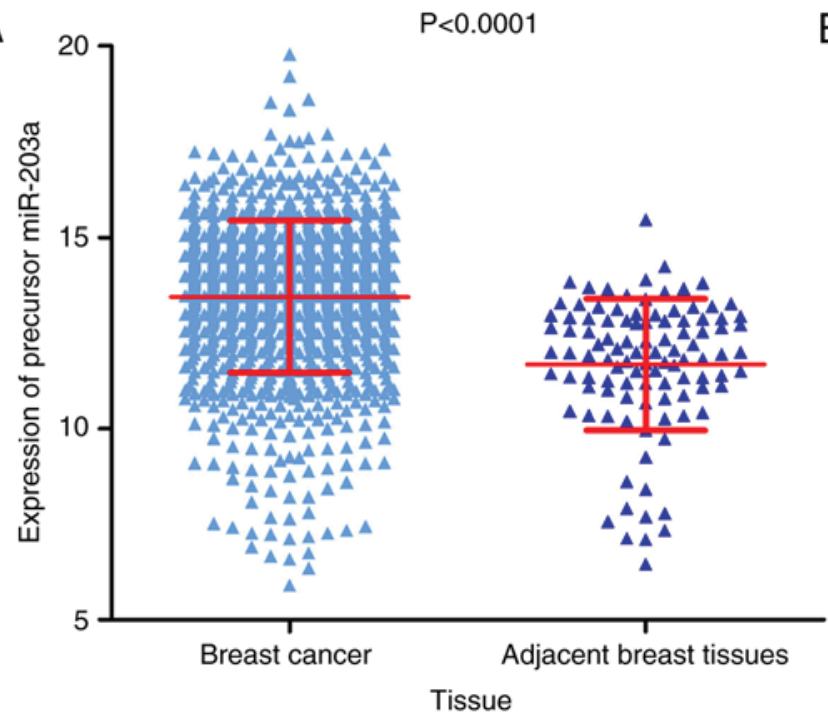

C

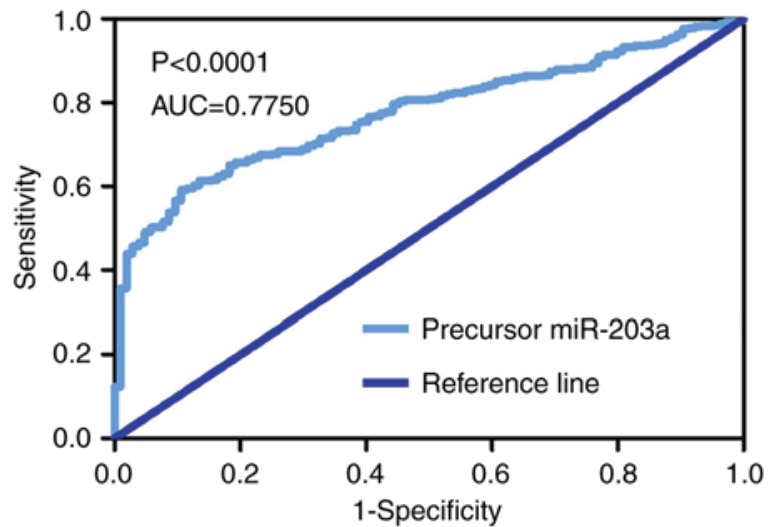

B

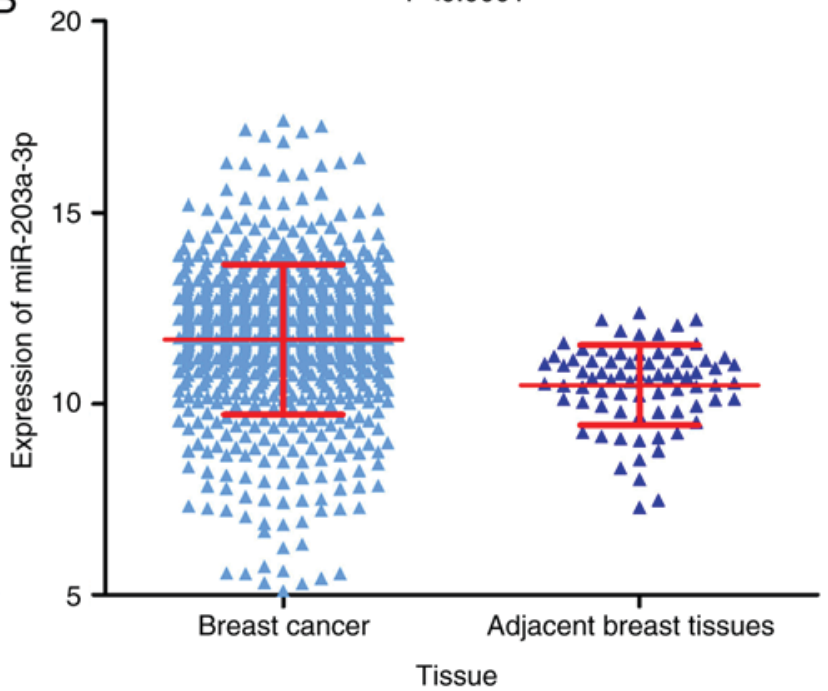

D

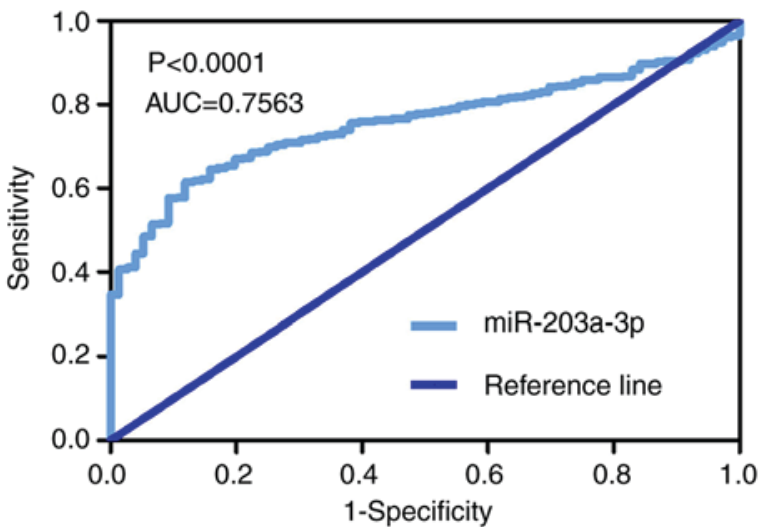

Figure 1. Clinical value of precursor miR-203a and miR-203a-3p in breast cancer tissue compared with adjacent breast tissue. (A) Expression of precursor miR-203a in breast cancer tissue compared with adjacent breast tissue based on TCGA data. (B) The expression of miR-203a-3p in breast cancer tissue compared with adjacent breast tissue based on UCSC Xena project. (C) ROC of precursor miR-203a in breast cancer tissue based on TCGA data. (D) ROC of miR-203a-3p in breast cancer tissue based on UCSC Xena project. miR, microRNA; TCGA, The Cancer Genome Atlas; UCSC, University of California Santa Cruz; ROC, receiver operating characteristic; AUC, area under the curve.

distinguish between $\mathrm{BC}$ tissue and adjacent breast tissue. It was also identified that the expression of precursor miR-203a was increased in three groups, including the $<60$ years old group, the negative ER group and the negative PR group, compared with their corresponding groups, the $\geq 60$ years old group, the positive ER group and the positive PR group (all $\mathrm{P}<0.05$; Fig. 2A-C, and Table I). The result of the survival analysis indicated that precursor miR-203a possessed no prognostic value in BC (Fig. 3).

Clinical value of miR-203a-3p in BC, using GEO data. Finally, nine GEO microarrays with 611 BC tissue samples and 379 adjacent breast tissue samples were selected for further analysis (Fig. 4). It was identified that the expression of miR-203a-3p was significantly upregulated in BC tissue compared with adjacent breast tissue in $3 \mathrm{GEO}$ microarrays (GSE37407, GSE40525 and GSE58606, all $\mathrm{P}<0.05$; Fig. 5). The ROC curve of these three microarrays also implied that miR-203a-3p could be used to distinguish between $\mathrm{BC}$ tissue and adjacent breast tissue (Fig. 6).
Meta-analysis. The result of SMD revealed that the expression of miR-203a-3p was markedly increased in 2,444 BC tissue cases compared with 559 adjacent breast tissue cases. The heterogeneity test indicated that there was significant heterogeneity in the included studies $\left(\mathrm{I}^{2}=91.5 \% ; \mathrm{P}=0.000\right.$; 95\% CI, 0.44-0.65; Fig. 7). Therefore, an influence analysis was conducted to seek the source of heterogeneity and it was identified that GSE44281 was significantly different from the other 10 studies (Fig. 8). Following the omission of GSE44281, the level of $\mathrm{I}^{2}$ was decreased, but still reached $86.9 \%$ (Fig. 9). The outcome of a funnel plot asymmetry test indicated that no publication bias was identified in the included studies (Fig. 10). The AUC of sROC reached 0.82 with a sensitivity of 0.70 (0.54-0.82) and a specificity of 0.81 (0.63-0.91), which implied that miR-203a-3p could be used to distinguish between BC tissue and adjacent breast tissue (Figs. 11 and 12).

GO enrichment, KEGG pathway analyses and PPI network. Online prediction tools were used to acquire a total of 4,565 predicted target genes, which had to appear at least four times in searches to qualify. Meanwhile, 2,669 DEGs from 
A

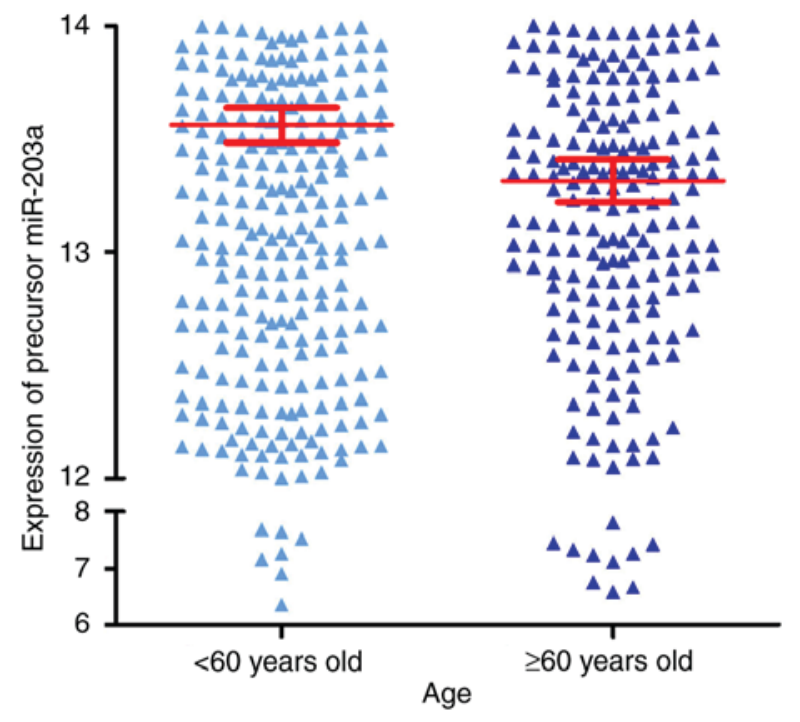

$P=0.0425$

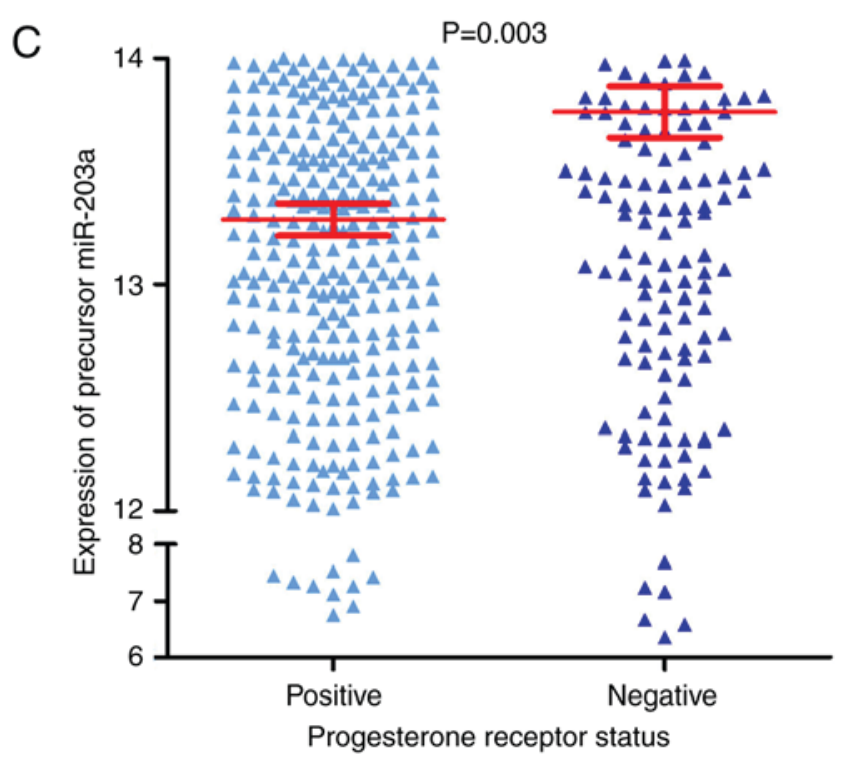

B

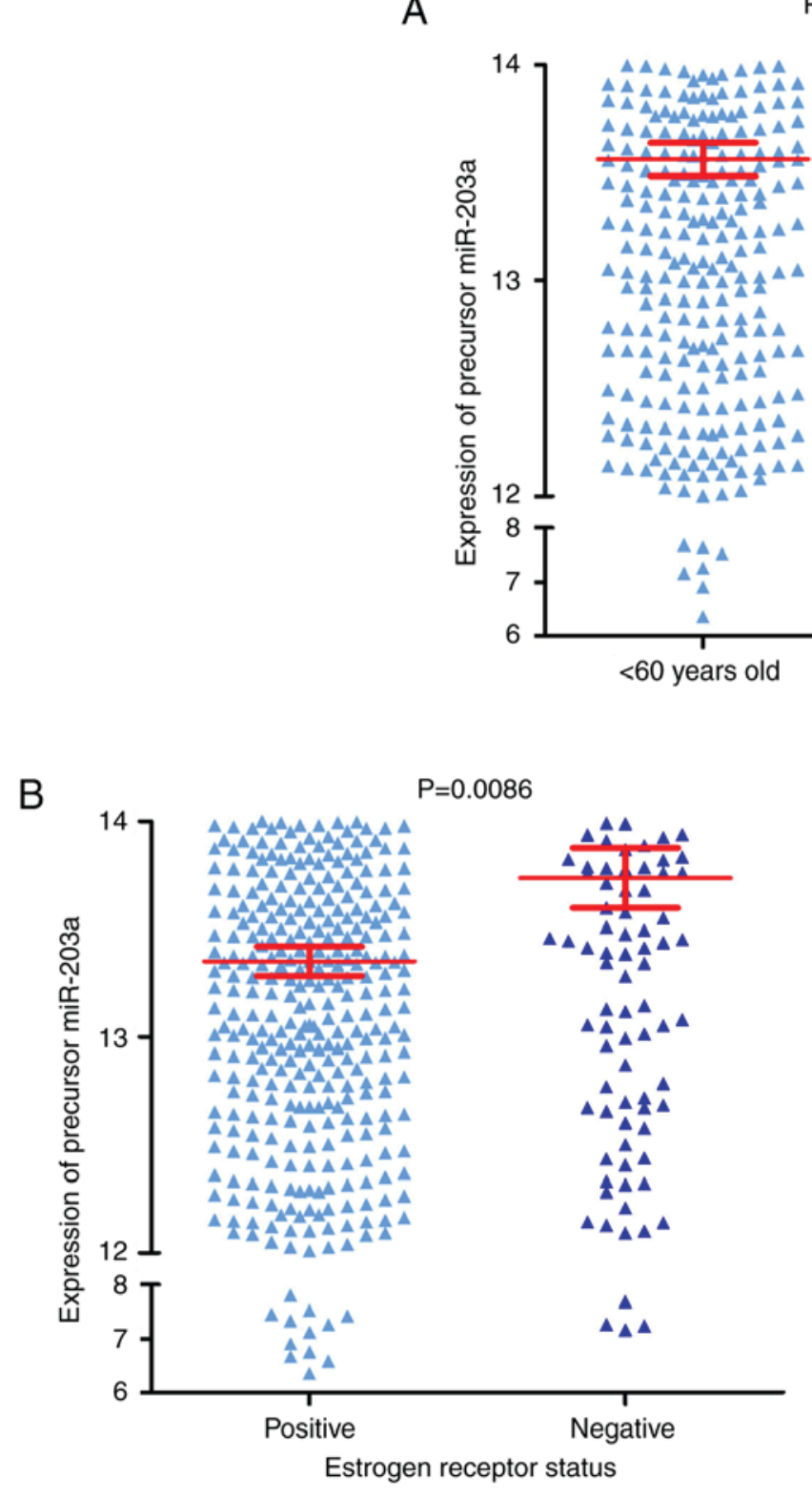

$\mathrm{P}=0.0086$

Figure 2. Expression of precursor miR-203a in groups divided by clinical parameters. (A) $\geq 60$ years vs. $<60$ years. (B) Positive estrogen receptor status vs. negative estrogen receptor status. (C) Positive progesterone receptor status vs. negative progesterone receptor status. miR, microRNA.

GEO and 2,138 DEGs from TCGA were acquired. Of these DEGs, 89 genes intersected with the predicted target genes (Fig. 13). The result of GO enrichment analysis indicated that the overlapped genes were associated with 'plasma membrane integrity', 'cell surface receptor linked signal transduction' and '3',5'-cyclic nucleotide phosphodiesterase activity' (Fig. 14; Table II). In addition, a pathway termed 'purine metabolism' was identified to be closely associated with miR-203a-3p expression in BC via its target genes, including phosphodiesterase $1 \mathrm{C}$ (PDE1C), adenylate cyclase 5 (ADCY5), phosphodiesterase 1A(PDE1A), phosphodiesterase 5A (PDE5A) and phosphodiesterase 8B (PDE8B; Table III). Notably, the expression of three genes (PDE1A, PDE1C and PDE8B) was significantly reduced in BC tissue compared with adjacent breast tissue. The other genes demonstrated a reduced trend in $\mathrm{BC}$ tissue compared to adjacent breast tissue, but no statistical significance was observed (Fig. 15).

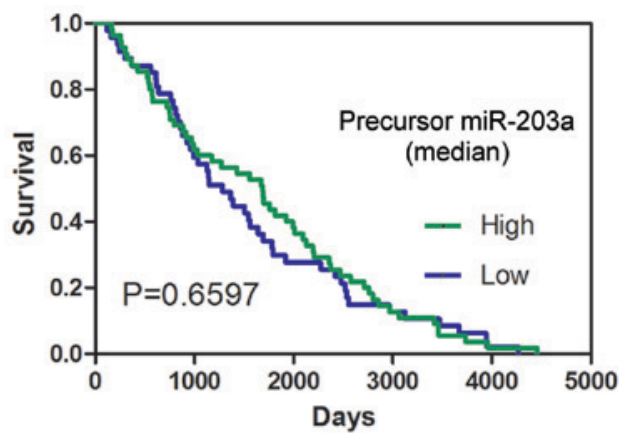

Figure 3. The survival curve of precursor miR-203a in breast cancer. The survival curve implied that miR-203a featured no prognosis value in breast cancer. miR, microRNA.

Spearman's correlation analysis identified that ADCY5 was negatively correlated with miR-203a-3p. A minor negative 
Table I. Expression of precursor miR-203a in groups divided from clinical parameters.

\begin{tabular}{|c|c|c|c|c|}
\hline \multirow[b]{2}{*}{ Clinical parameters } & \multirow[b]{2}{*}{$\mathrm{n}$} & \multicolumn{3}{|c|}{ miR-203a expression } \\
\hline & & Mean \pm standard deviation & $\mathrm{T}$ or $\mathrm{F}$ & P-value \\
\hline Tissue & & & $9.83^{\mathrm{a}}$ & $<0.001^{\mathrm{c}}$ \\
\hline Normal & 104 & $11.69 \pm 1.72$ & & \\
\hline Breast cancer & 1,077 & $13.45 \pm 1.97$ & & \\
\hline Age (years) & & & $-2.01^{\mathrm{a}}$ & $0.045^{\mathrm{c}}$ \\
\hline$\leq 60$ & 592 & $13.56 \pm 1.88$ & & \\
\hline$>60$ & 485 & $13.32 \pm 2.08$ & & \\
\hline Sex & & & $-1.10^{\mathrm{a}}$ & 0.295 \\
\hline Female & 1,065 & $13.46 \pm 1.98$ & & \\
\hline Male & 12 & $12.91 \pm 1.71$ & & \\
\hline Vital status & & & $-0.84^{\mathrm{a}}$ & 0.401 \\
\hline Alive & 975 & $13.43 \pm 1.98$ & & \\
\hline Dead & 102 & $13.60 \pm 1.89$ & & \\
\hline Pathologic stage & & & $\mathrm{F}=1.253^{\mathrm{b}}$ & \\
\hline Stage I & 181 & $13.44 \pm 1.65$ & & 0.289 \\
\hline Stage II & 609 & $13.46 \pm 2.11$ & & \\
\hline Stage III & 244 & $13.50 \pm 1.89$ & & \\
\hline Stage IV & 20 & $12.61 \pm 1.89$ & & \\
\hline $\mathrm{T}$ & & & $\mathrm{F}=0.707^{\mathrm{b}}$ & \\
\hline $\mathrm{T} 1$ & 279 & $13.54 \pm 1.72$ & & 0.548 \\
\hline $\mathrm{T} 2$ & 620 & $13.45 \pm 2.07$ & & \\
\hline $\mathrm{T} 3$ & 135 & $12.27 \pm 2.00$ & & \\
\hline $\mathrm{T} 4$ & 40 & $13.26 \pm 2.16$ & & \\
\hline $\mathrm{N}$ & & & $-1.14^{\mathrm{a}}$ & \\
\hline No & 508 & $13.37 \pm 2.00$ & & 0.254 \\
\hline Yes & 549 & $13.51 \pm 1.96$ & & \\
\hline M & & & $1.81^{\mathrm{a}}$ & \\
\hline No & 893 & $13.44 \pm 1.94$ & & 0.085 \\
\hline Yes & 21 & $12.69 \pm 1.87$ & & \\
\hline Estrogen receptor status & & & $-2.63^{\mathrm{a}}$ & \\
\hline Positive & 795 & $13.35 \pm 1.91$ & & $0.009^{\mathrm{c}}$ \\
\hline Negative & 232 & $13.74 \pm 2.13$ & & \\
\hline Progesterone receptor status & & & $-3.52^{\mathrm{a}}$ & \\
\hline Positive & 689 & $13.29 \pm 1.88$ & & $<0.001^{\mathrm{c}}$ \\
\hline Negative & 335 & $13.76 \pm 2.09$ & & \\
\hline HER2 status & & & $0.60^{\mathrm{a}}$ & \\
\hline Positive & 164 & $13.55 \pm 0.15$ & & 0.549 \\
\hline Negative & 564 & $13.45 \pm 0.08$ & & \\
\hline
\end{tabular}

${ }^{\mathrm{a}}$ T-test was applied. ${ }^{\mathrm{b}}$ One-way analysis of variance was applied. ${ }^{\mathrm{c}} \mathrm{P}<0.05$ was considered to indicate a statistically significant difference. $\mathrm{T}, \mathrm{N}$ and $\mathrm{M}$ based on TNM staging. miR, microRNA; HER2, human epidermal growth factor receptor 2.

correlation was identified between the other four genes and miR-203a-3p, but no statistical significance was observed (Fig. 16). The ROC demonstrated that all these genes could be used to distinguish between BC tissue and adjacent breast tissue (Fig. 17). Through the PPI network, four hub genes were identified: Epidermal growth factor receptor, ADCY5, metalloproteinase inhibitor 3 and insulin-like growth factor 1 (IGF1; Fig. 18). Depending on data from TCGA, it was identified that only IGF1 was predominantly decreased in BC tissue compared with adjacent breast tissue (Figs. 15A, 19 and 20A). As the expression of the hub genes should be decreased in BC tissue compared with adjacent breast tissue, IGF1 was identified as the hub gene of miR-203a-3p in BC. Furthermore, IGF1 exhibited a distinction between BC tissue 


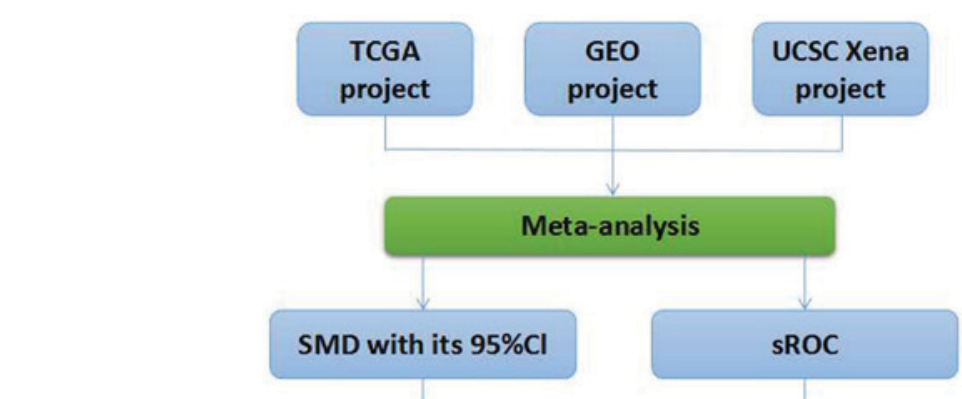

\section{Expression level and potential molecular mechanism of miR-203a-3p in breast cancer}

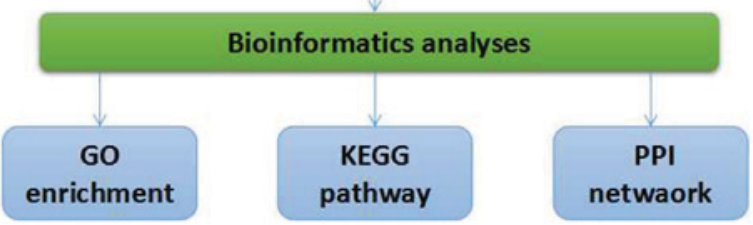

Figure 4. Flow chart of the present study. TCGA, The Cancer Genome Atlas; GEO, Gene Expression Omnibus; UCSC, University of California Santa Cruz; SMD, standard mean difference; CI, confidence interval; sROC, summarized receiver operating characteristic; miR, microRNA; GO, gene ontology; KEGG, Kyoto Encyclopedia of Genes and Genomes; PPI, protein-protein interaction.
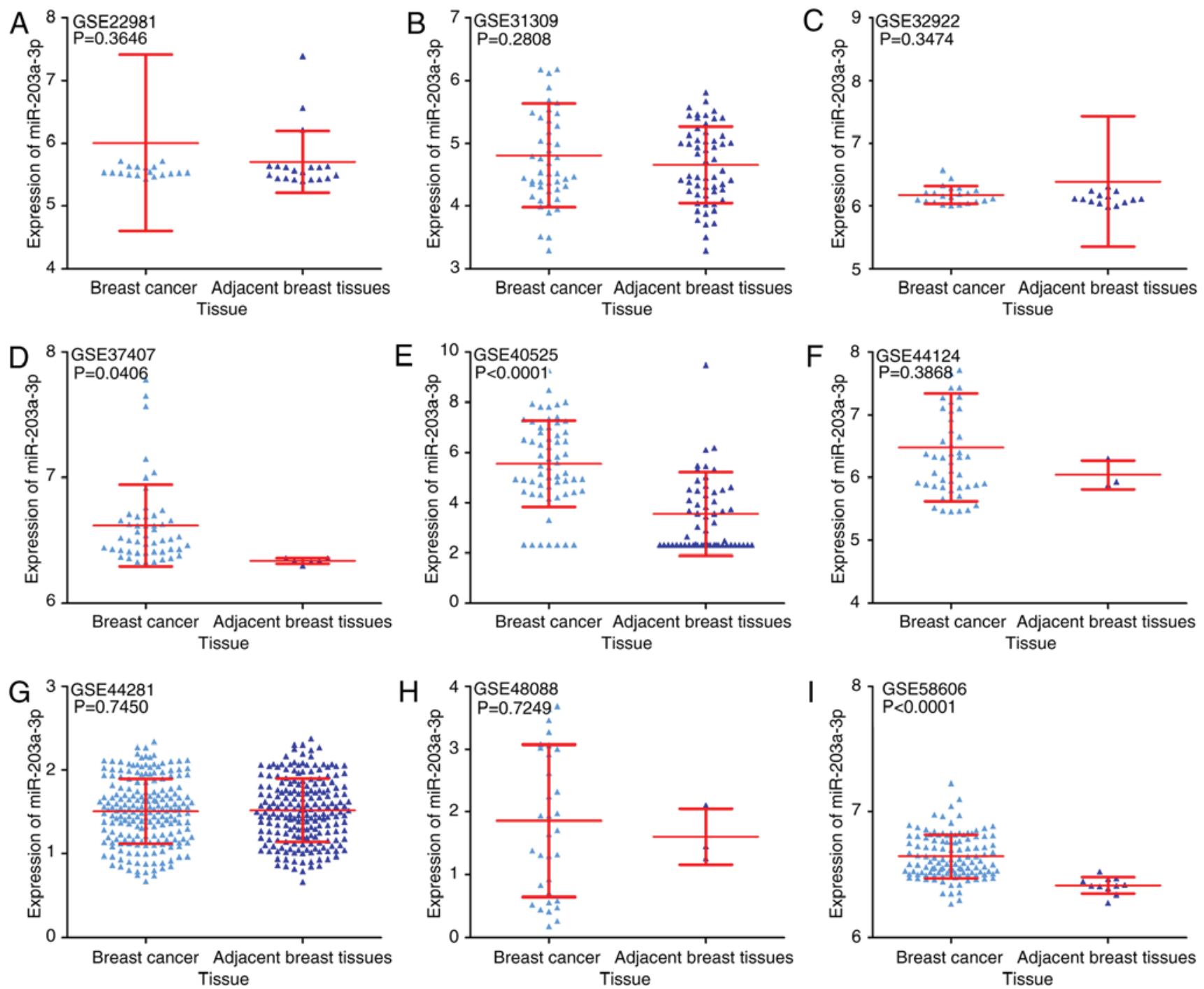

Figure 5. Expression of miR-203a-3p in breast cancer tissue and adjacent breast tissue in Gene Expression Omnibus datasets. (A) GSE22981, (B) GSE31309, (C) GSE32922, (D) GSE37407, (E) GSE40525, (F) GSE44124, (G) GSE44281, (H) GSE48088 and (I) GSE58606. miR, microRNA. 

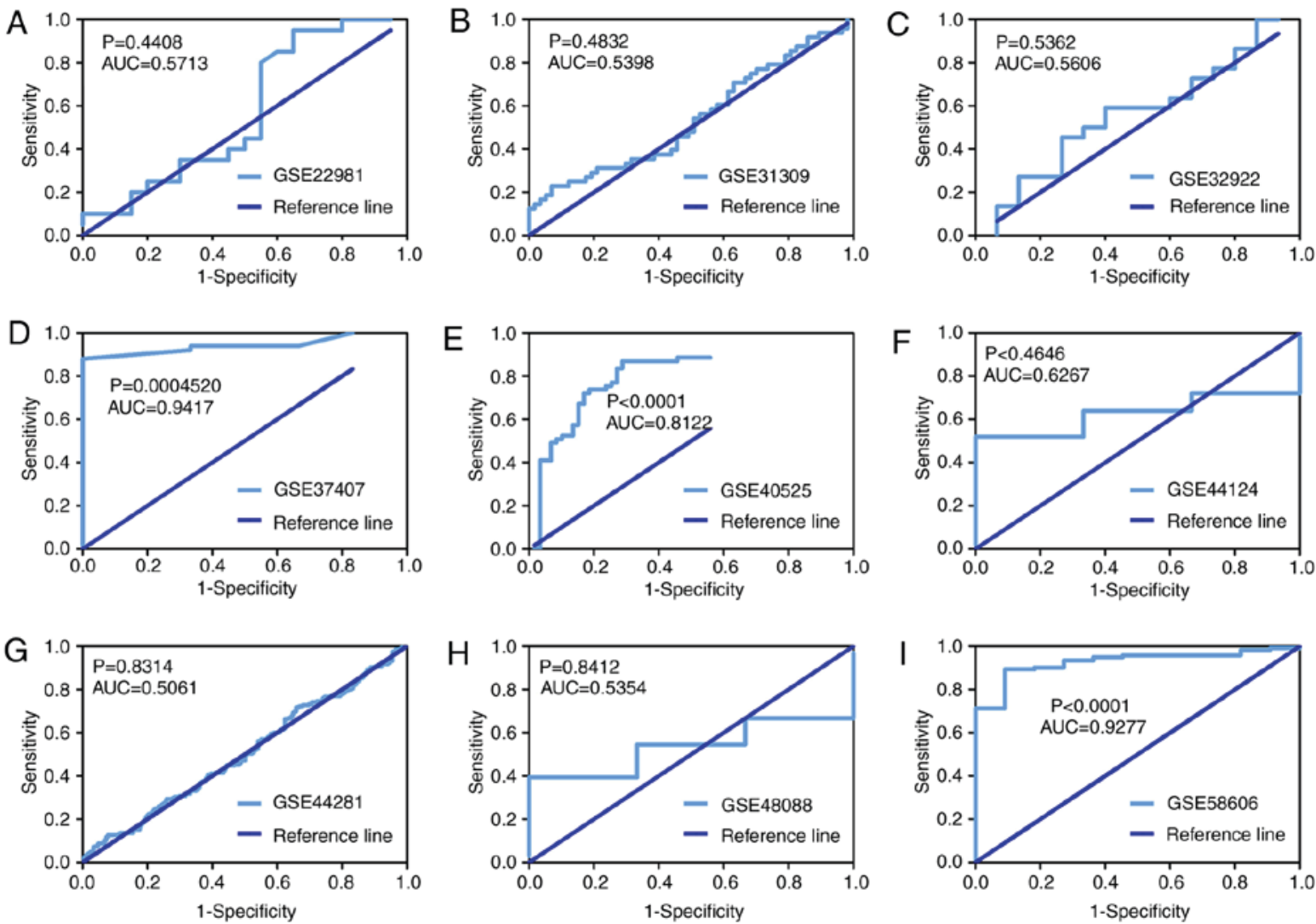

Figure 6. Receiver operating characteristics of microRNA-203a-3p in breast cancer tissue and adjacent breast tissue in Gene Expression Omnibus datasets. (A) GSE22981, (B) GSE31309, (C) GSE32922, (D) GSE37407, (E) GSE40525, (F) GSE44124, (G) GSE44281, (H) GSE48088 and (I) GSE58606. AUC, area under the curve.

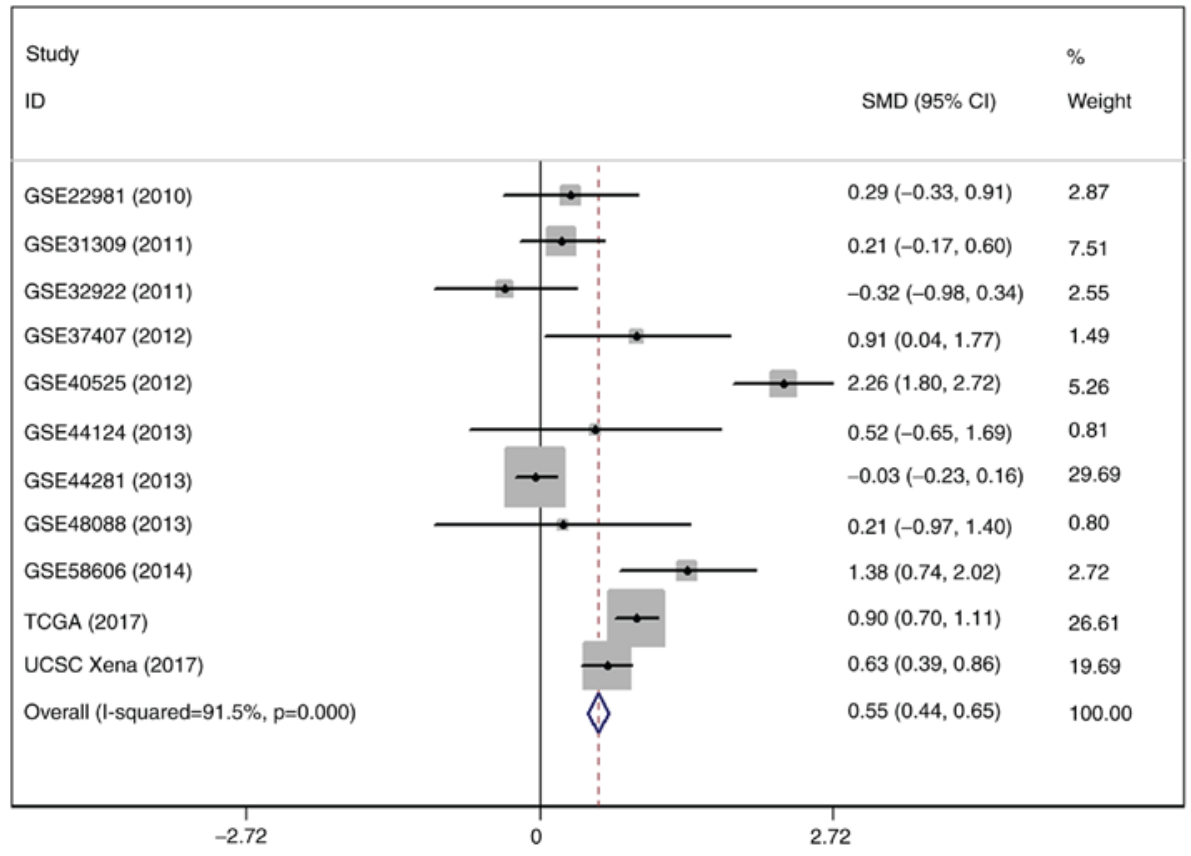

Figure 7. Forest plot to evaluate the expression of miR-203a-3p in breast cancer tissue and adjacent breast tissue prior to deleting GSE44281 (2013). The expression of miR-203a-3p was markedly increased in breast cancer tissue compared to adjacent breast tissue. miR, microRNA; SMD, standard mean difference; CI, confidence interval; TCGA, The Cancer Genome Atlas; UCSC, University of California Santa Cruz.

and adjacent breast tissue with an AUC of ROC that reached 0.9348 (Fig. 20B). Additionally, a slight negative correlation was identified between IGF1 and miR-203a-3p according to the Spearman's correlation analysis; however, the correlation was not statistically significant $(r=-0.1611 ; P=0.4038$; Fig. 20C). 


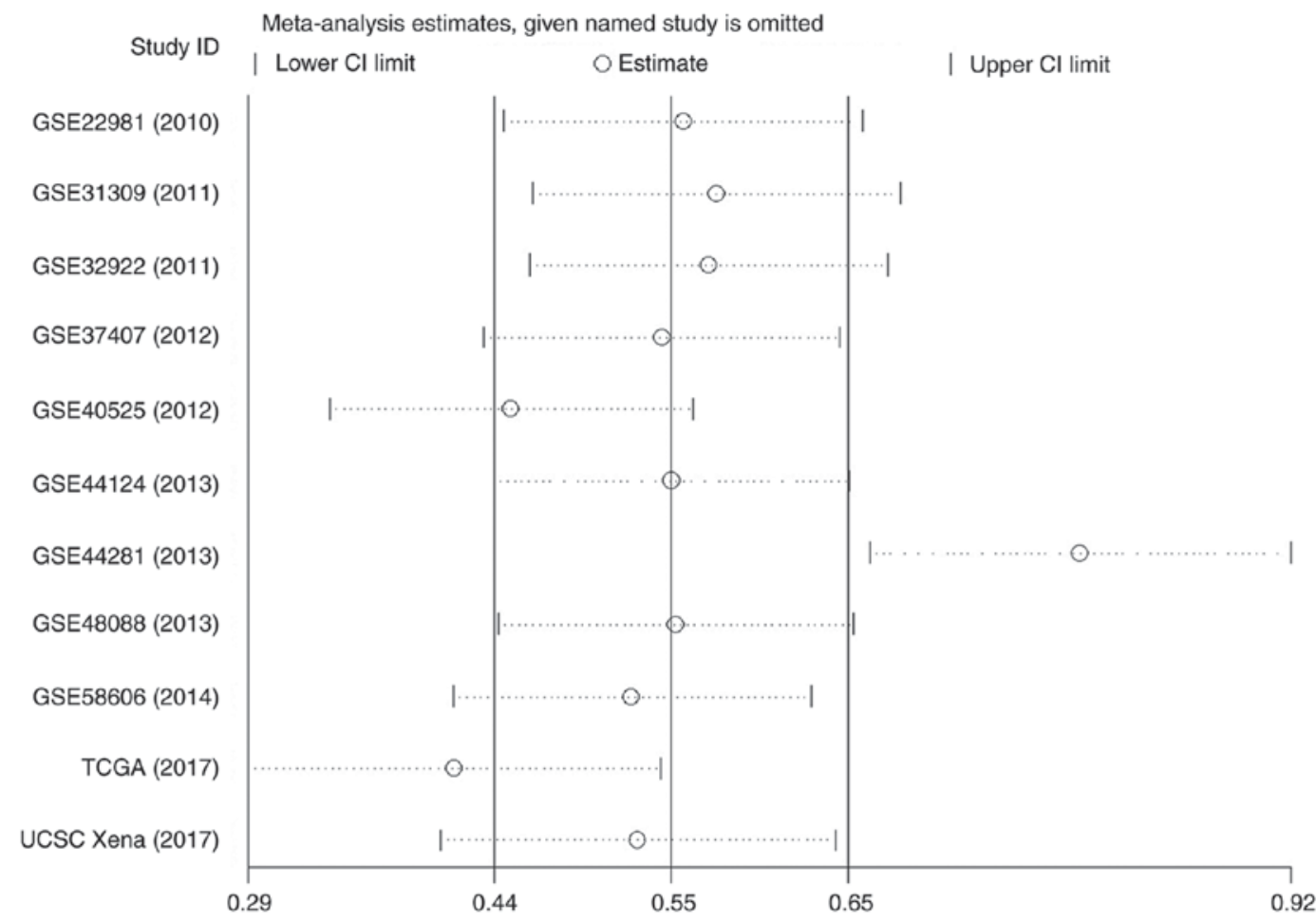

C1

Figure 8. Influence analysis of the included studies. GSE44281 (2013) was substantially different from the other 10 studies. CI, confidence interval; TCGA, The Cancer Genome Atlas; UCSC, University of California Santa Cruz.

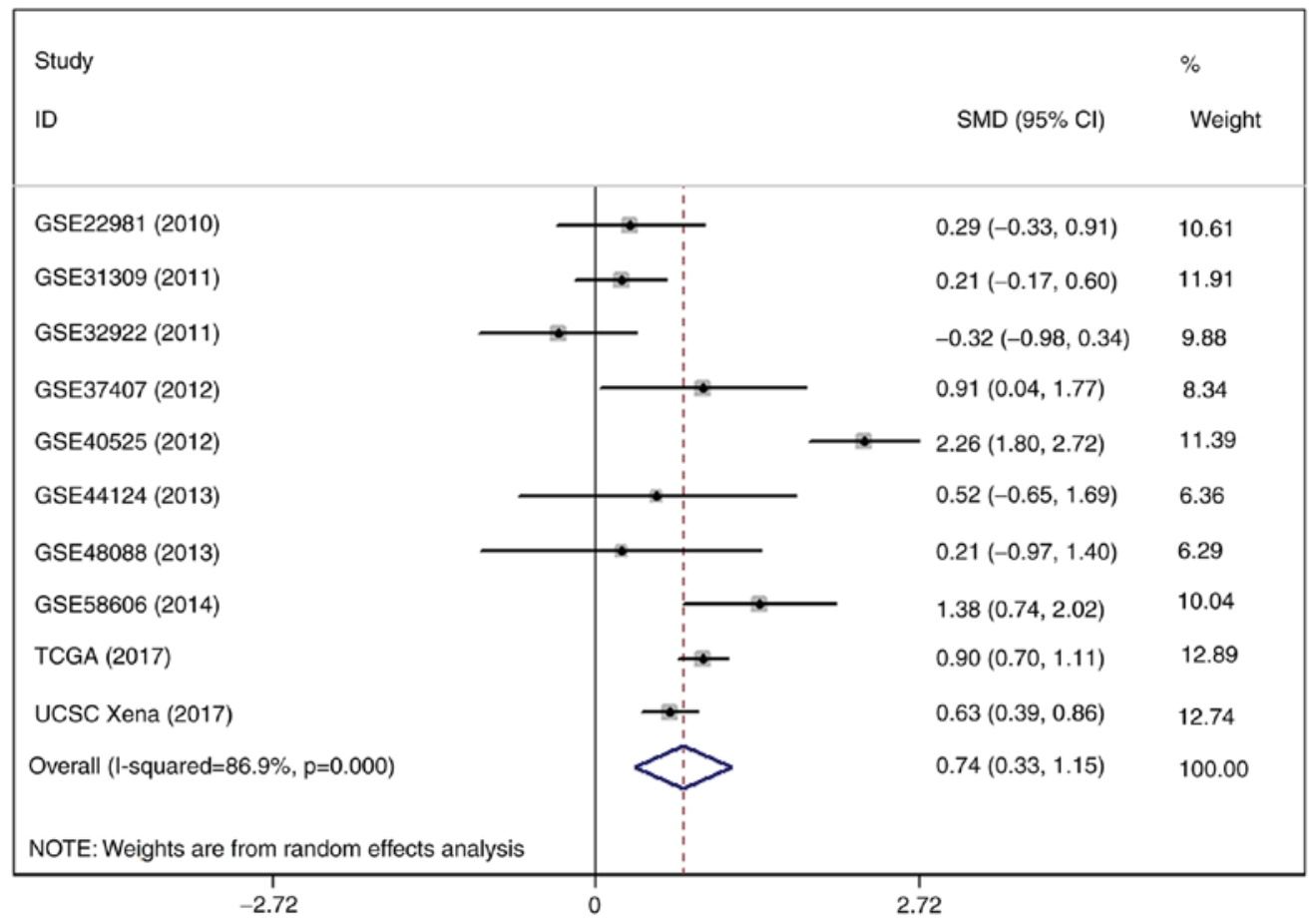

Figure 9. Forest plot to evaluate the expression of miR-203a-3p in breast cancer tissue and adjacent breast tissue following deleting GSE44281 (2013). The expression of miR-203a-3p was markedly increased in breast cancer tissue compared with adjacent breast tissue. miR, microRNA; SMD, standard mean difference; CI, confidence interval; TCGA, The Cancer Genome Atlas; UCSC, University of California Santa Cruz.

\section{Discussion}

Previous studies have identified that miR-203a-3p is significantly associated with various cancers; a trend of miR-203a-3p elevation has been detected in hepatocellular (33) and colorectal (34) carcinoma. By contrast, downregulated miR-203a-3p expression was detected in gastric cancer (35), prostate cancer (36), non-small-cell lung carcinoma (37) and esophageal cancer (38). 
Table II. GO enrichment of the 89 overlapped genes.

\begin{tabular}{|c|c|c|c|c|}
\hline GO ID & Term & Count & Ontology & P-value \\
\hline GO:0007166 & Cell surface receptor linked signal transduction & 22 & $\mathrm{BP}$ & $3.877 \times 10^{-4}$ \\
\hline GO:0007167 & Enzyme linked receptor protein signaling pathway & 8 & $\mathrm{BP}$ & $2.061 \times 10^{-3}$ \\
\hline GO:0019932 & Second-messenger-mediated signaling & 6 & $\mathrm{BP}$ & $7.787 \times 10^{-3}$ \\
\hline GO:0030335 & Positive regulation of cell migration & 4 & BP & $1.140 \times 10^{-2}$ \\
\hline GO:0030334 & Regulation of cell migration & 5 & BP & $1.194 \times 10^{-2}$ \\
\hline GO:0009725 & Response to hormone stimulus & 7 & $\mathrm{BP}$ & $1.247 \times 10^{-2}$ \\
\hline GO:0007242 & Intracellular signaling cascade & 14 & $\mathrm{BP}$ & $1.281 \times 10^{-2}$ \\
\hline GO:0050806 & Positive regulation of synaptic transmission & 3 & $\mathrm{BP}$ & $1.367 \times 10^{-2}$ \\
\hline GO:0040017 & Positive regulation of locomotion & 4 & $\mathrm{BP}$ & $1.477 \times 10^{-2}$ \\
\hline GO:0051272 & Positive regulation of cell motion & 4 & $\mathrm{BP}$ & $1.477 \times 10^{-2}$ \\
\hline GO:0005887 & Integral to plasma membrane & 16 & $\mathrm{CC}$ & $6.200 \times 10^{-4}$ \\
\hline GO:0031226 & Intrinsic to plasma membrane & 16 & $\mathrm{CC}$ & $7.800 \times 10^{-4}$ \\
\hline GO:0044459 & Plasma membrane part & 22 & $\mathrm{CC}$ & $1.800 \times 10^{-3}$ \\
\hline GO:0016021 & Integral to membrane & 37 & $\mathrm{CC}$ & $1.200 \times 10^{-2}$ \\
\hline GO:0044433 & Cytoplasmic vesicle part & 5 & $\mathrm{CC}$ & $1.400 \times 10^{-2}$ \\
\hline GO:0005886 & Plasma membrane & 28 & $\mathrm{CC}$ & $2.100 \times 10^{-2}$ \\
\hline GO:0031224 & Intrinsic to membrane & 37 & $\mathrm{CC}$ & $2.100 \times 10^{-2}$ \\
\hline GO:0031091 & Platelet $\alpha$ granule & 3 & $\mathrm{CC}$ & $3.200 \times 10^{-2}$ \\
\hline GO:0030659 & Cytoplasmic vesicle membrane & 4 & $\mathrm{CC}$ & $3.200 \times 10^{-2}$ \\
\hline GO:0012506 & Vesicle membrane & 4 & $\mathrm{CC}$ & $4.000 \times 10^{-2}$ \\
\hline GO:0004114 & 3',5'-cyclic-nucleotide phosphodiesterase activity & 4 & MF & $3.300 \times 10^{-4}$ \\
\hline GO:0004112 & Cyclic-nucleotide phosphodiesterase activity & 4 & MF & $3.740 \times 10^{-4}$ \\
\hline GO:0008081 & Phosphoric diester hydrolase activity & 5 & MF & $1.330 \times 10^{-3}$ \\
\hline GO:0004117 & Calmodulin-dependent cyclic-nucleotide phosphodiesterase activity & 2 & MF & $1.700 \times 10^{-2}$ \\
\hline GO:0003690 & Double-stranded DNA binding & 4 & MF & $1.788 \times 10^{-2}$ \\
\hline GO:0003779 & Actin binding & 6 & $\mathrm{MF}$ & $3.819 \times 10^{-2}$ \\
\hline GO:0008144 & Drug binding & 3 & MF & $4.182 \times 10^{-2}$ \\
\hline GO:0043566 & Structure-specific DNA binding & 4 & MF & $4.981 \times 10^{-2}$ \\
\hline GO:0004714 & Transmembrane receptor protein tyrosine kinase activity & 3 & MF & $5.588 \times 10^{-2}$ \\
\hline GO:0060090 & Molecular adaptor activity & 3 & MF & $5.588 \times 10^{-2}$ \\
\hline
\end{tabular}

GO, gene ontology; BP, biological process; CC, cellular component; MF, molecular function.

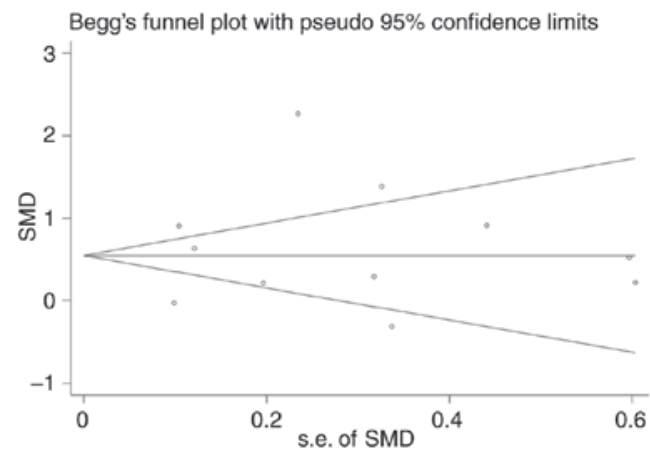

Figure 10. The funnel plot indicated that no publication bias was identified. s.e., standard error; SMD, standard mean difference.

However, only one study has identified the expression and potential functions of miR-203a-3p in BC; Gomes et al (21) reported that the expression of miR-203a-3p was markedly upregulated in $109 \mathrm{BC}$ samples compared with matched normal

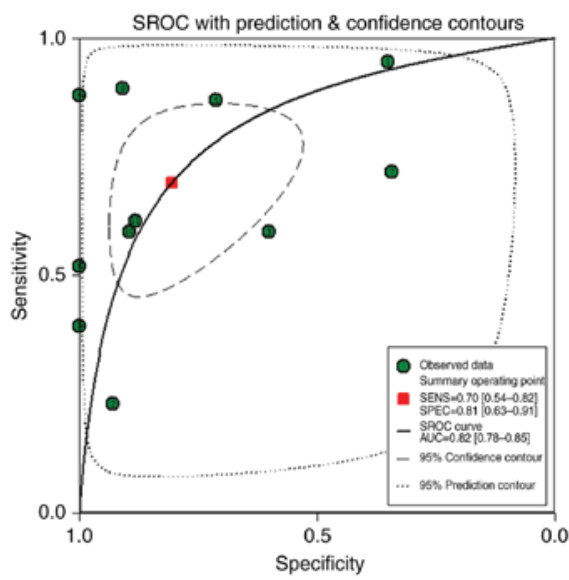

Figure 11. sROC of microRNA-203a-3p in breast cancer based on data from The Cancer Genome Atlas, Gene Expression Omnibus and University of California Santa Cruz Xena projects. The AUC was 0.82 , indicating an apparent distinguishability between breast cancer tissue and adjacent breast tissue. sROC, summarized receiver operating characteristic; SENS, sensitivity; SPEC, specificity; AUC, area under the curve. 

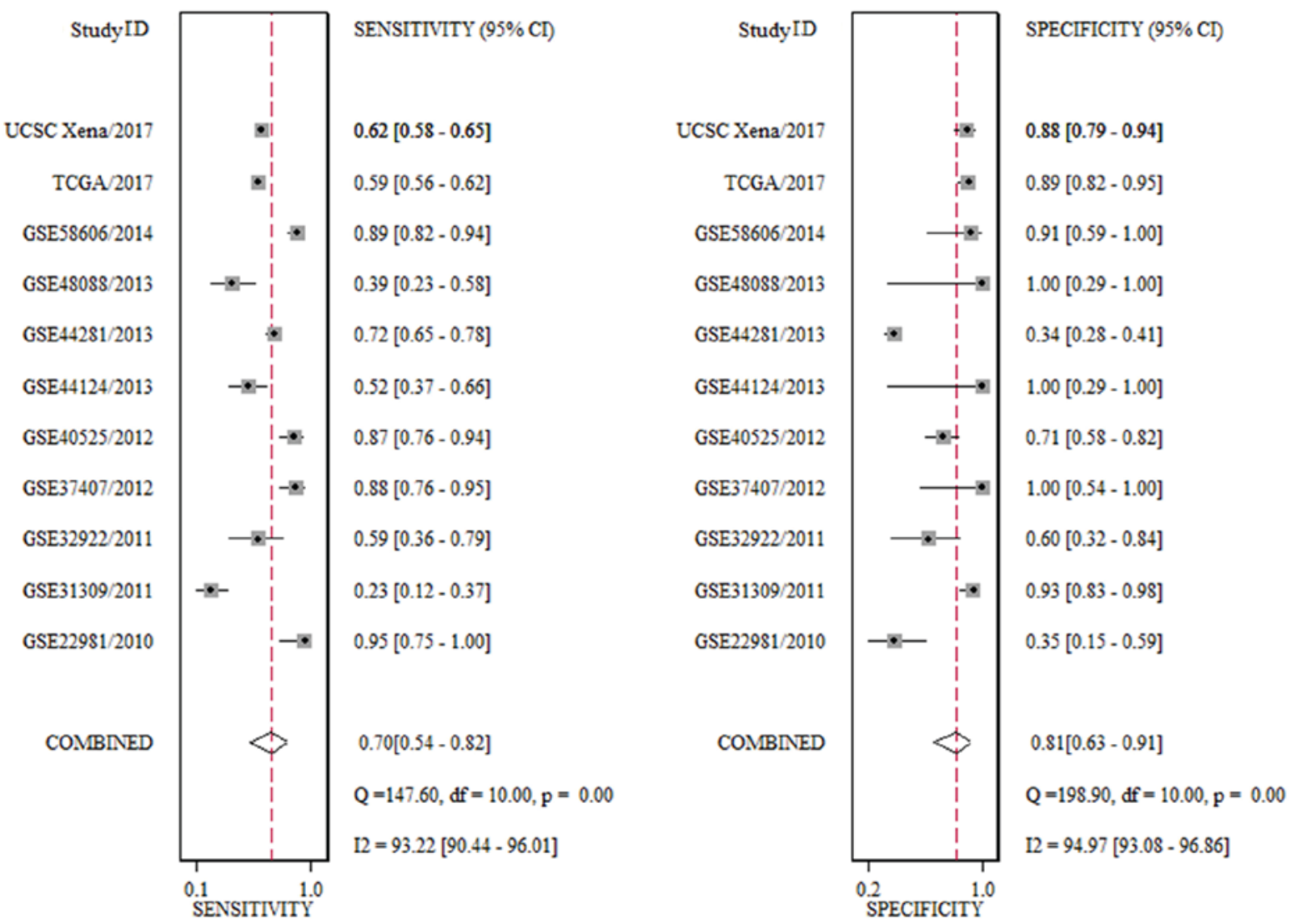

Figure 12 . The sensitivity and specificity of sROC. The sensitivity and specificity of sROC were $0.70(0.54-0.82)$ and $0.81(0.63-0.91)$, respectively. sROC, summarized receiver operating characteristic; CI, confidence interval; UCSC, University of California Santa Cruz; TCGA, The Cancer Genome Atlas.

breast samples and also identified that upregulated expression of miR-203a-3p was established in five clinic pathological characteristics groups: Tumor size $\leq 18.5 \mathrm{~mm}$, HER2-negative, PR-positive, ER-positive and high Ki-67 index groups.

Since the sample size of the study by Gomes et al (21) was not large or varied enough, the current study combined data from three projects with a larger sample size to ensure the accuracy of the results. It was identified that the expression of precursor miR-203a was significantly elevated in 1,077 BC tissue samples compared with 104 adjacent breast tissue samples in TCGA project data. In the UCSC Xena project, the expression of miR-203a-3p was significantly increased in 756 BC tissue cases compared with 76 adjacent breast tissue cases. In addition, an elevated trend was detected in $\mathrm{BC}$ tissues compared with adjacent breast tissue in three GEO microarrays. The outcome of the comprehensive meta-analysis indicated that the expression of miR-203a-3p trended toward overexpression in 2,444 BC tissue cases compared with 559 adjacent breast tissue cases. Additionally, ROC and sROC suggested that miR-203a-3p could be used to distinguish between $\mathrm{BC}$ tissue and adjacent breast tissue. It was detected that upregulated miR-203a-3p was associated with age ( $<60$-year-old patients), PR-negative BC tissue and ER-negative $\mathrm{BC}$ tissue. Regarding the prognosis value of miR-203a-3p in $\mathrm{BC}$, no prognostic value was observed. Taken together, it was hypothesized that miR-203a-3p may enhance the development and oncogenesis of $\mathrm{BC}$.

GO enrichment and KEGG pathway analyses were conducted to identify the potential molecular mechanism of the role of miR-203a-3p in BC. The predicted miR-203a-3p

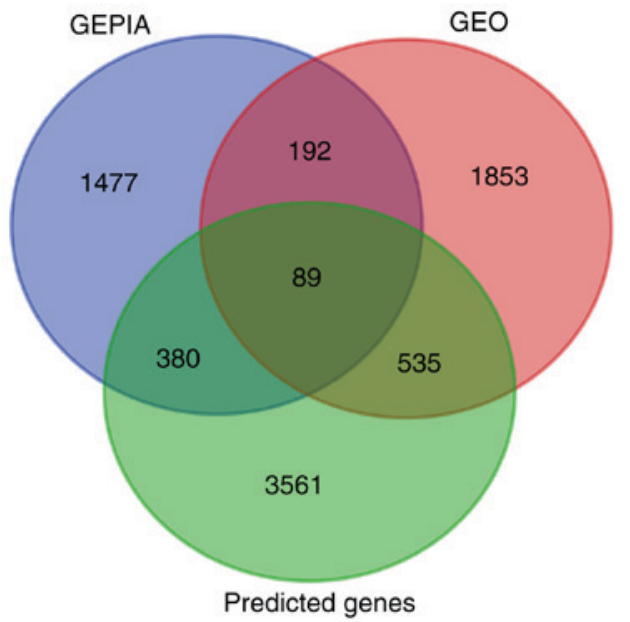

Figure 13. Venn diagram showing the intersection of differentially expressed genes from GEO, GEPIA and predicted target genes. In all, 89 overlapped genes were identified. GEPIA, gene expression profiling interactive analysis; GEO, Gene Expression Omnibus.

target genes were significantly enriched in three biological processes: 'Plasma membrane', 'cell surface receptor linked signal transduction' and ' 3 ',5'-cyclic nucleotide phosphodiesterase activity'. Therefore, it was hypothesized that miR-203a-3p may influence BC via the above processes. In addition, a pathway termed 'purine metabolism' was closely associated with miR-203a-3p target genes. The expression and ROCs of the pathway-related genes were assessed; the 


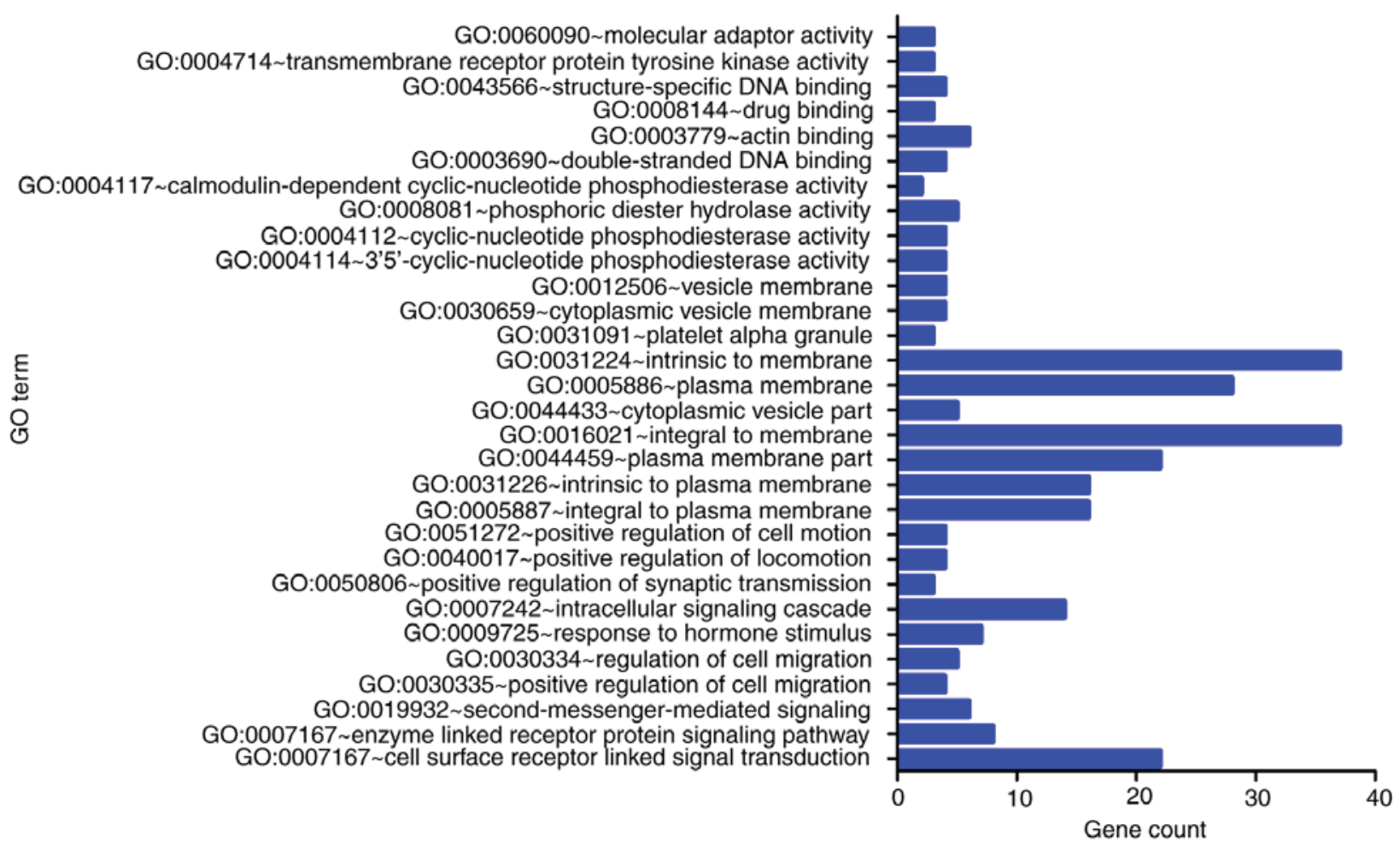

Figure 14. GO enrichment of the 89 overlapped genes. The 89 overlapped genes were correlated with plasma membrane integrity, cell surface receptor linked signal transduction and 3',5'-cyclic nucleotide phosphodiesterase activity. GO, gene ontology.
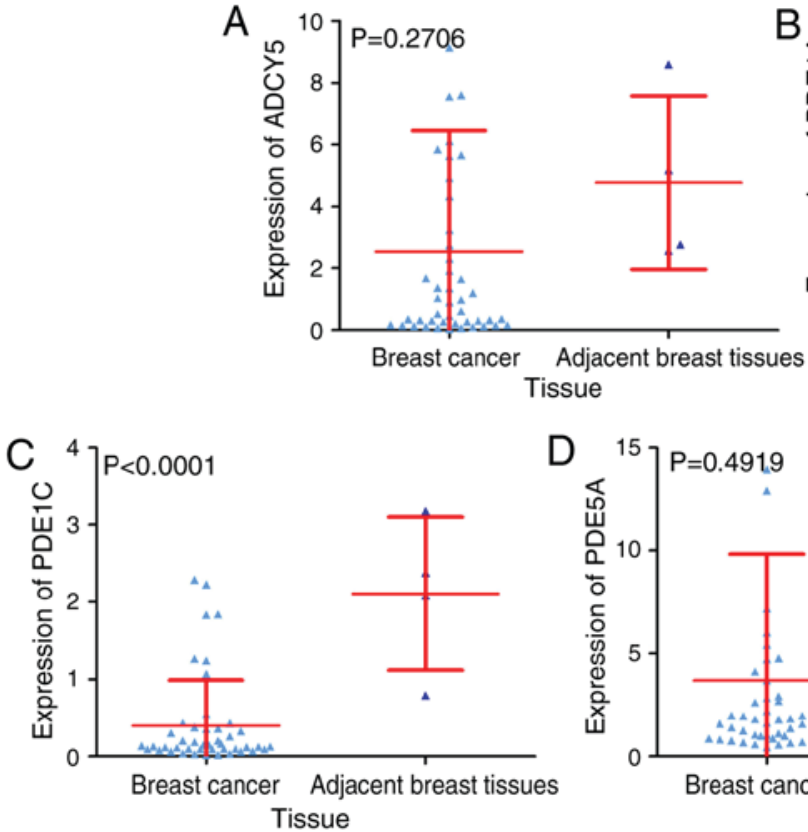

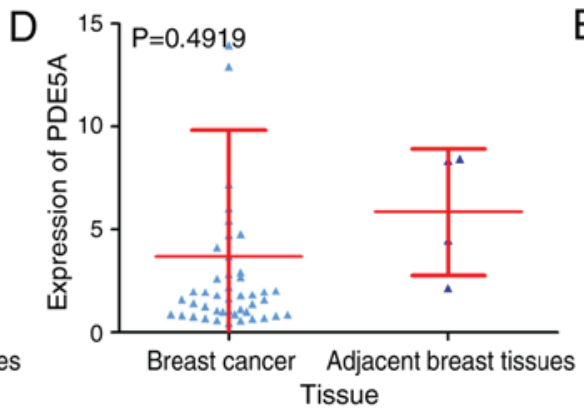

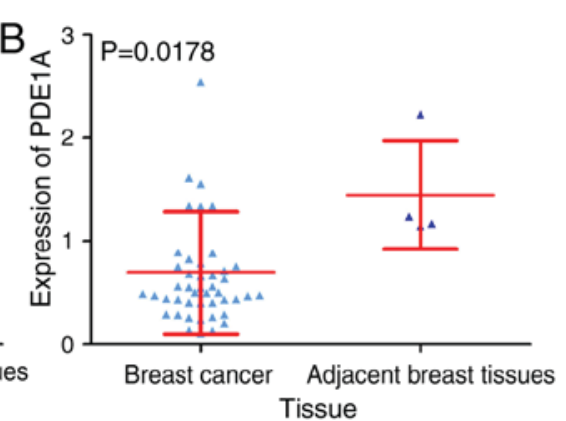

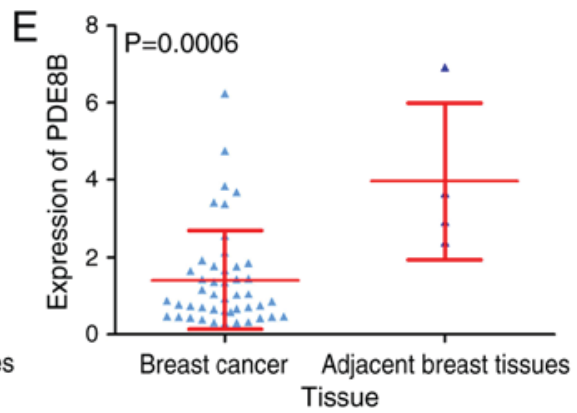

Figure 15. Expression of genes in the purine metabolism pathway. (A) ADCY5, (B) PDE1A, (C) PDE1C, (D) PDE5A and (E) PDE8B. ADCY5, adenylate cyclase 5; PDE1A, phosphodiesterase 1A; PDE1C, phosphodiesterase 1C; PDE5A, phosphodiesterase 5A; PDE8B, phosphodiesterase 8B.

expression of three genes (PDE1C, PDE1A and PDE8B) was significantly decreased in $\mathrm{BC}$ tissue compared with adjacent breast tissue and the expression of other genes (PDE5A and ADCY5) was marginally reduced in $\mathrm{BC}$ tissue compared with adjacent breast tissue, but the change was not statistically significant. ROCs from these five genes indicated that each was able to distinguish $\mathrm{BC}$ from adjacent normal tissue. In addition, it was detected that ADCY5 expression was negatively correlated with miR-203a-3p expression. Taken together, the findings indicate that miR-203a-3p may be involved in purine metabolism in $\mathrm{BC}$ by targeting ADCY5, PDE1C, PDE1A, PDE5A and PDE8B.

Finally, the hub gene IGF1 was selected for further investigation. IGF1 is regarded as a vital gene in regulating 
Table III. KEGG pathway of the 89 overlapped genes.

\begin{tabular}{llll}
\hline ID & \multicolumn{1}{c}{ Term } & Count & P-value \\
\hline hsa00230: & Purine metabolism & 5 & $9.189 \times 10^{-3}$ \\
hsa04020: & Calcium signaling pathway & 5 & $1.483 \times 10^{-2}$ \\
hsa05214: & Glioma & 3 & $4.645 \times 10^{-2}$ \\
hsa05218: & Melanoma & 3 & $5.755 \times 10^{-2}$ \\
hsa05212: & Pancreatic cancer & 3 & $5.899 \times 10^{-2}$ \\
hsa04914: & Progesterone-mediated oocyte maturation & 3 & $8.051 \times 10^{-2}$ \\
hsa05215: & Prostate cancer & 3 & $8.540 \times 10^{-2}$ \\
hsa04666: & Fc $\gamma$ R-mediated phagocytosis & 3 & $9.545 \times 10^{-2}$ \\
hsa00230: & Purine metabolism & 5 & $9.189 \times 10^{-3}$
\end{tabular}

KEGG, Kyoto Encyclopedia of Genes and Genomes.
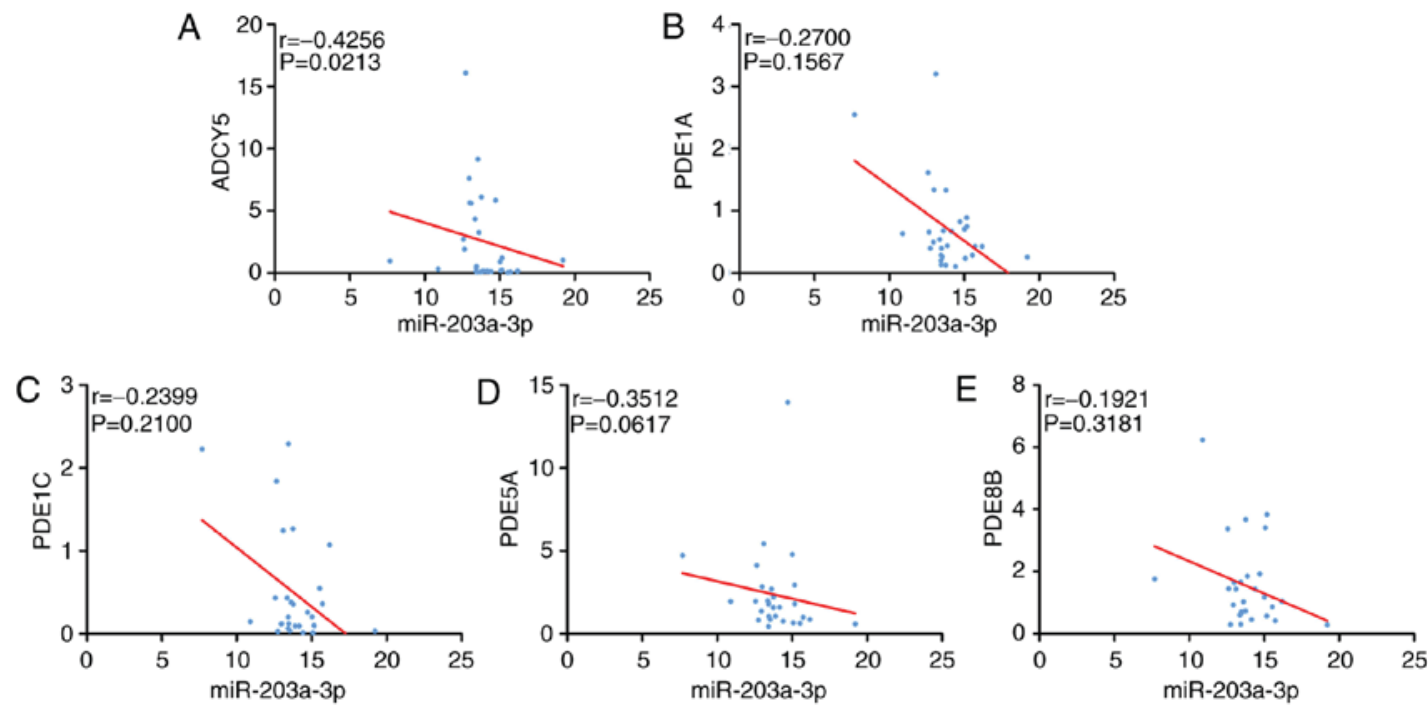

Figure 16. The correlation between pathway-associated genes and miR-203a-3p based on The Cancer Genome Atlas project data. (A) ADCY5. (B) PDE1A. (C) PDE1C. (D) PDE5A. (E) PDE8B. ADCY5, adenylate cyclase 5; PDE1A, phosphodiesterase 1A; PDE1C, phosphodiesterase 1C; PDE5A, phosphodiesterase 5A; PDE8B, phosphodiesterase 8B; miR, microRNA.
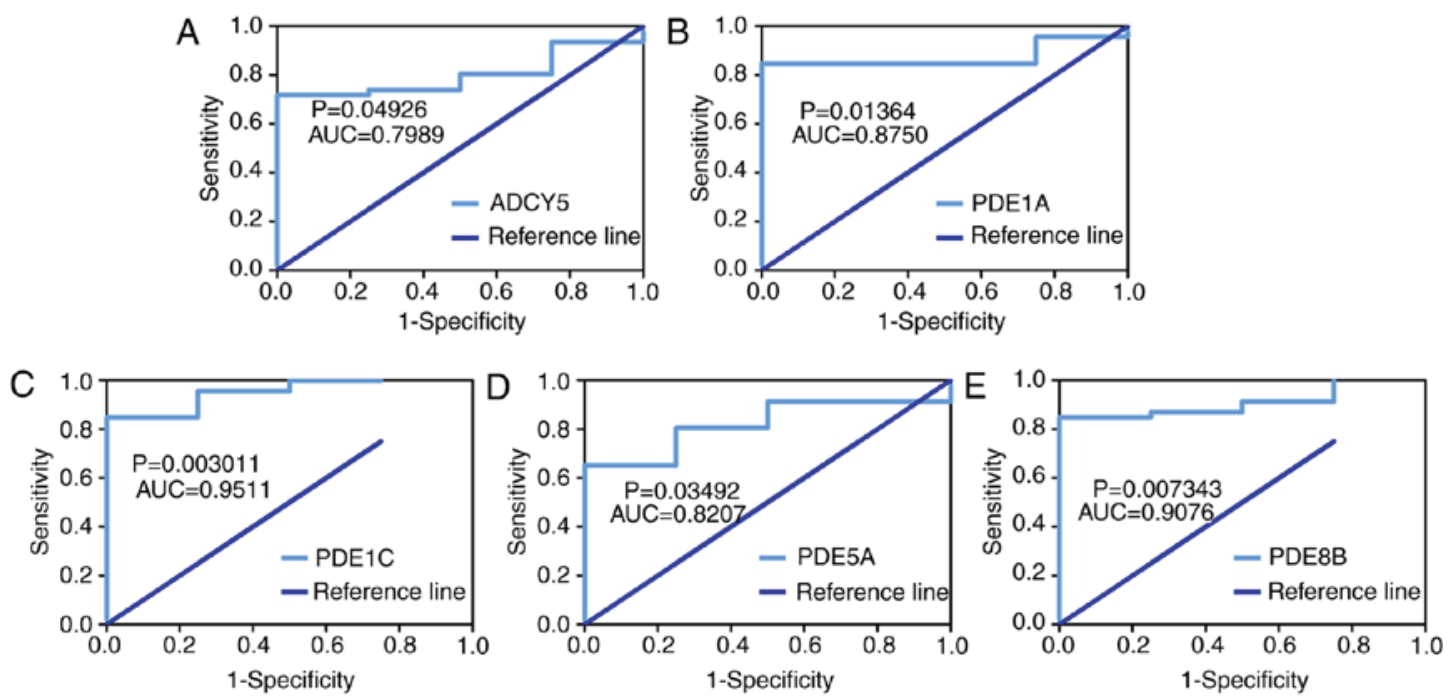

Figure 17. Receiver operating characteristic of genes in 'purine metabolism' pathway. (A) ADCY5, (B) PDE1A, (C) PDE1C, (D) PDE5A and (E) PDE8B. AUC, area under the curve; ADCY5, adenylate cyclase 5; PDE1A, phosphodiesterase 1A; PDE1C, phosphodiesterase 1C; PDE5A, phosphodiesterase 5A; PDE8B, phosphodiesterase $8 \mathrm{~B}$. 


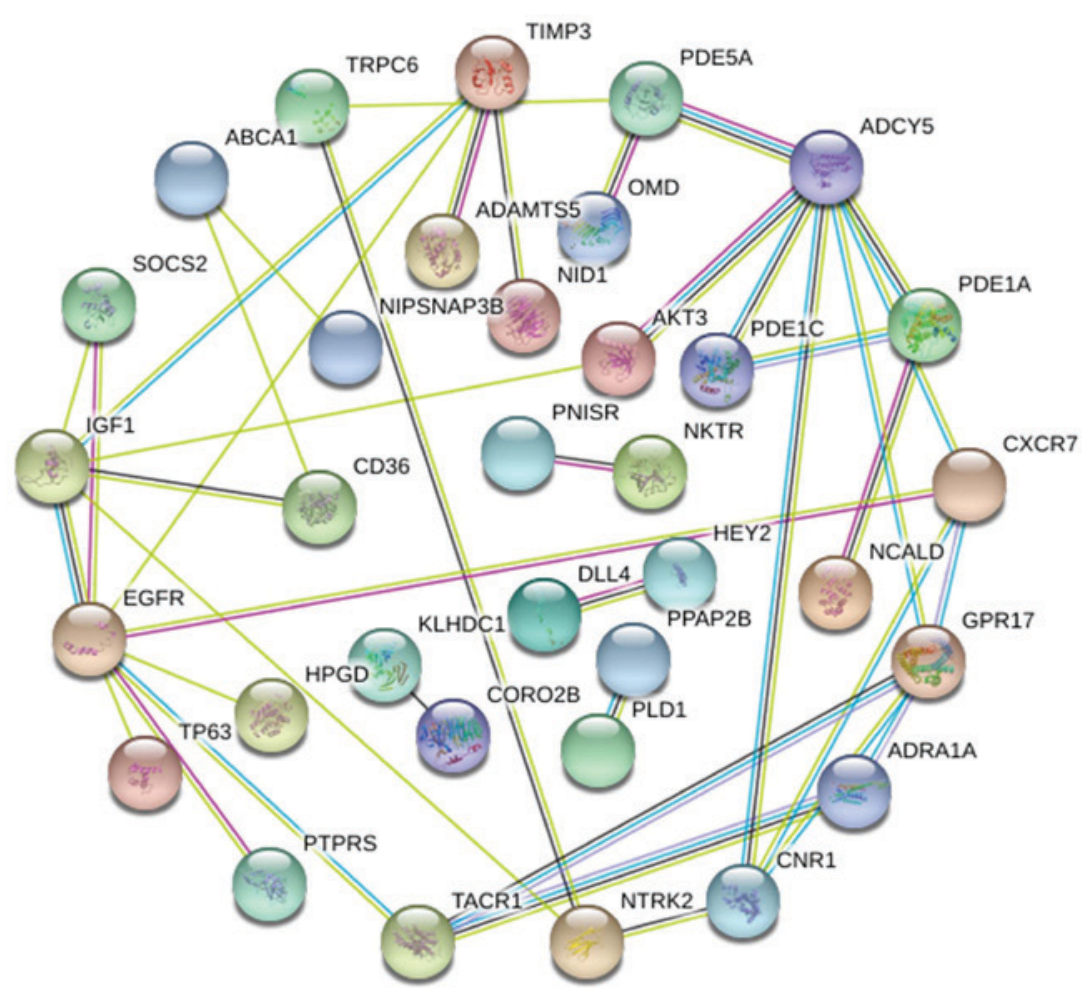

Figure 18. The protein-protein interaction network of miR-203a-3p in breast cancer. IGF1 was identified as the hub gene of miR-203a-3p in breast cancer. Each node represents a different gene. The line color indicates the type of evidence for interaction. The magenta line and blue lines signify known interaction; the green line signifies predicted interaction; the black line and purple line signify other interactions. miR, microRNA; IGF1, insulin-like growth factor 1.
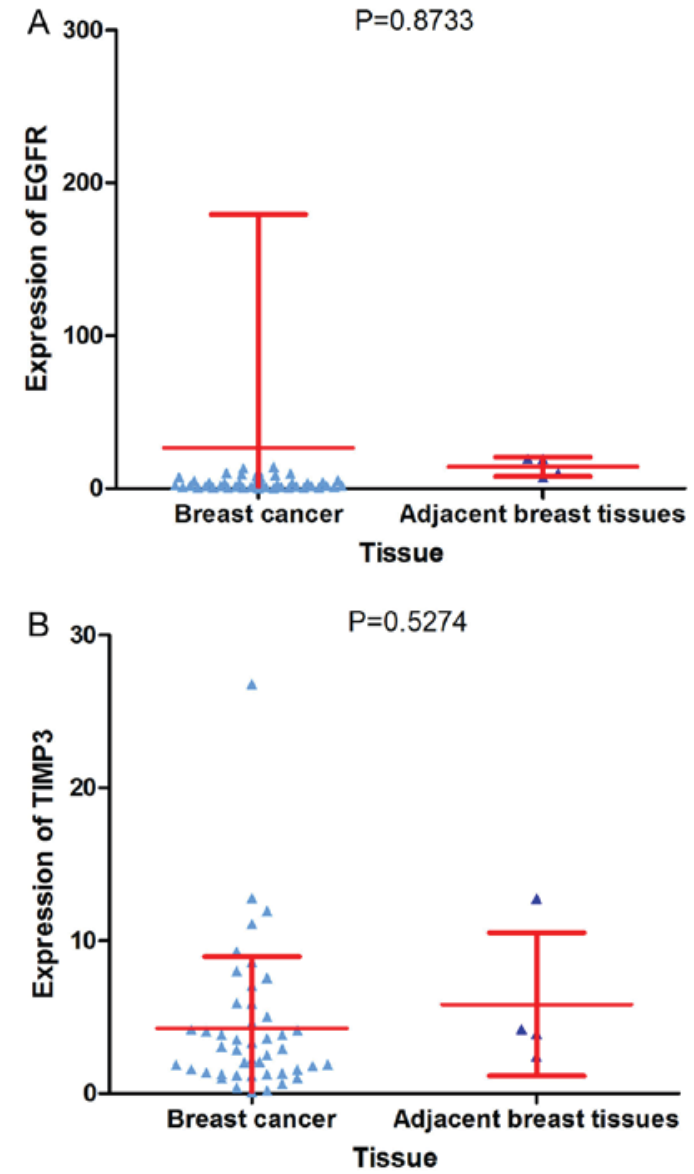

Figure 19. The expression of EGFR and TIMP3 based on The Cancer Genome Atlas project. (A) EGFR and (B) TIMP3. EGFR, epidermal growth factor receptor; TIMP3, metalloproteinase inhibitor 3. cell differentiation, apoptosis and proliferation in $\mathrm{BC}$. IGF1 polymorphisms may enhance the risk for BC (39). De Santi et al (40) demonstrated that IGF1 is comprised a pro-form and mature form. The IGF1 pro-form enhances cell proliferation in BC via IGF1 receptor. The current study evaluated the expression and diagnostic ability of IGF1 in $\mathrm{BC}$ tissue and adjacent breast tissue. It was identified that the expression of IGF1 was reduced in BC tissue compared with adjacent breast tissue and IGF1 could be used to distinguish $\mathrm{BC}$ tissue; however, the negative correlation between IGF1 and miR-203a-3p expression was not statistically significant. The findings of the present study suggested that miR-203a-3p may be involved in certain pivotal processes in BC by targeting IGF1.

Although certain findings were acquired from the comprehensive meta-analysis and bioinformatics analyses, there are limitations of the current study. The heterogeneity test indicated that there was significant heterogeneity in the included studies; although an attempt was made to solve this. Unfortunately, the level of $\mathrm{I}^{2}$ was still $>50 \%$ following the omission of the source of heterogeneity. It was hypothesized that the following factors may have resulted in the significant heterogeneity: i) The GEO microarrays were acquired from different countries with four microarrays obtained from Spain (GSE32922, GSE44124, GSE48088 and GSE58606), two microarrays obtained from USA (GSE22981, GSE44281) and GSE31309, GSE37407 and GSE40525 were acquired from Germany, Sweden and Israel, respectively; ii) the approaches for determining the expression of miR-203a-3p were different across the different studies. Various platforms were conducted to analyze GEO microarrays. Furthermore, 

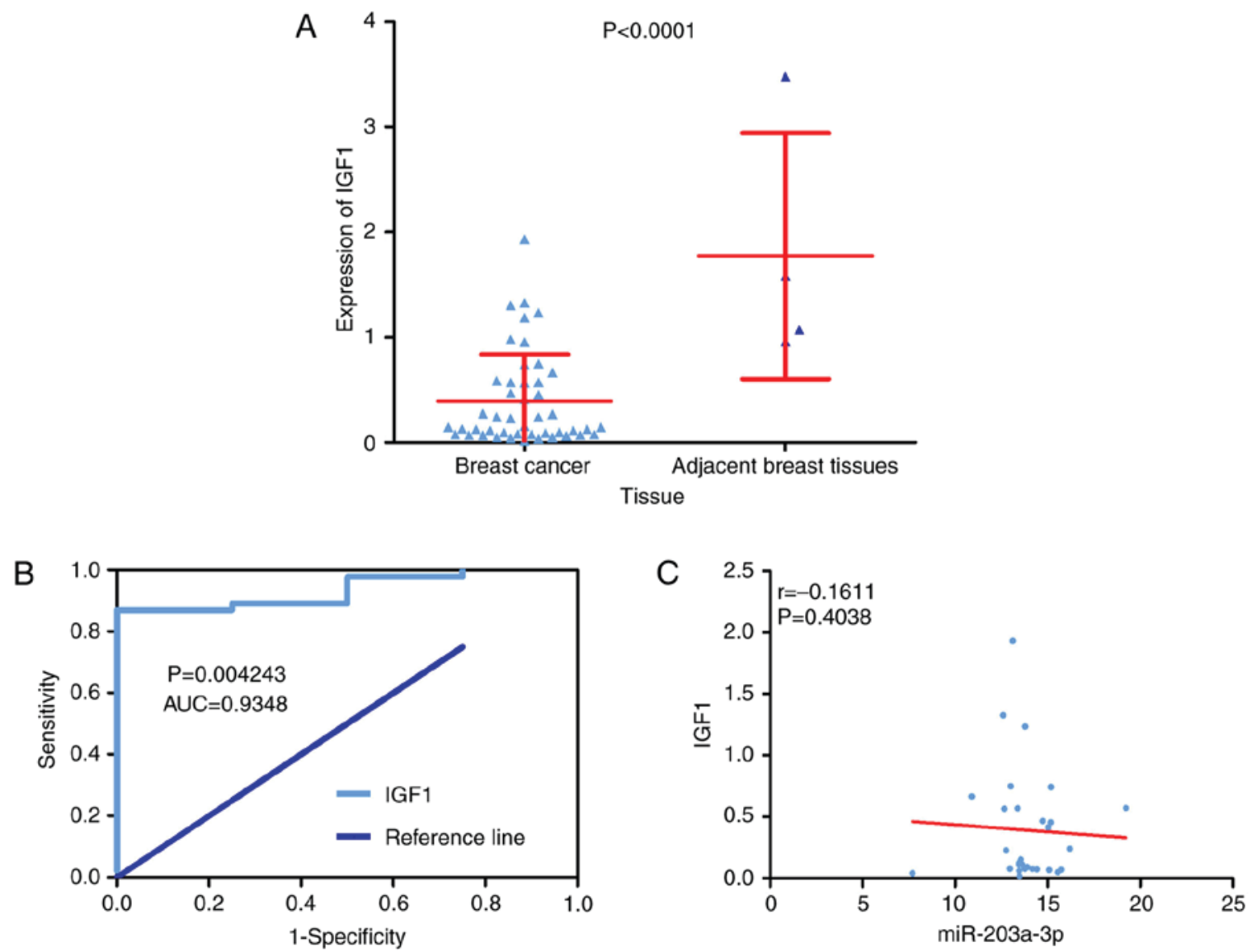

Figure 20. Clinical relevance of IGF1 in breast cancer. (A) The expression of IGF1 was markedly reduced in breast cancer tissue compared with adjacent breast tissue. (B) Receiver operating characteristics of IGF1 in breast cancer tissue. (C) Correlation between IGF1 and miR-203a-3p. miR, microRNA; AUC, area under the curve; IGF1, insulin-like growth factor 1.

in vitro or in vivo experiments to support the hypothesis of the present study were not performed, which is a major limitation. Thus, in vitro and in vivo studies should be performed in the near future.

In general, the present study established that the expression of miR-203a-3p was markedly elevated in BC tissue compared with adjacent breast tissue. Thus, it is hypothesized that miR-203a-3p may enhance the development and oncogenesis of BC. In addition, the target gene IGF1 was identified as a hub gene of miR-203a-3p in BC while the expression of IGF1 was significantly reduced in $\mathrm{BC}$ tissue compared with adjacent breast tissue.

\section{Acknowledgements}

Not applicable.

\section{Funding}

The present study was funded by Guangxi Zhuang Autonomous Region University Student Innovative Plan (grant no. 201710598065).

\section{Availability of data and materials}

The data and materials of the present study are available from the corresponding authors on reasonable request.

\section{Authors' contributions}

CF, JZha, RH and JM collected and analyzed the data. KC and JZho conceived the study and wrote the manuscript. All authors read the final version of the manuscript.

\section{Ethics approval and consent to participate}

Not applicable.

\section{Patient consent for publication}

Not applicable.

\section{Competing interests}

The authors declare that they have no competing interests.

\section{References}

1. Jalali C, Ghaderi B, Amini S, Abdi M and Roshani D: Association of XRCC1 Trp194 allele with risk of breast cancer, and Ki67 protein status in breast tumor tissues. Saudi Med J 37: 624-630, 2016.

2. Liang $\mathrm{Z}$ and Xi Y: MicroRNAs mediate therapeutic and preventive effects of natural agents in breast cancer. Chin J Nat Med 14: 881-887, 2016.

3. Siegel RL, Miller KD and Jemal A: Cancer statistics, 2018. CA Cancer J Clin 68: 7-30, 2018. 
4. Shou D, Wen L, Song Z, Yin J, Sun Q and Gong W: Suppressive role of myeloid-derived suppressor cells (MDSCs) in the microenvironment of breast cancer and targeted immunotherapies. Oncotarget 7: 64505-64511, 2016.

5. Xing L, He Q, Wang YY, Li HY and Ren GS: Advances in the surgical treatment of breast cancer. Chin Clin Oncol 5: 34, 2016.

6. Hennequin C, Barillot I, Azria D, Belkacémi Y Bollet M, Chauvet B, Cowen D, Cutuli B, Fourquet A, Hannoun-Lévi JM, et al: Radiotherapy of breast cancer. Cancer Radiother 20 (Suppl): S139-S146, 2016 (In French).

7. Ejlertsen B: Adjuvant chemotherapy in early breast cancer. Dan Med J 63: B5222, 2016.

8. Zhang X, Ren D, Guo L, Wang L, Wu S, Lin C, Ye L, Zhu J, Li J, Song L, et al: Thymosin beta 10 is a key regulator of tumorigenesis and metastasis and a novel serum marker in breast cancer. Breast Cancer Res 19: 15, 2017.

9. Li Z and Kang Y: Emerging therapeutic targets in metastatic progression: A focus on breast cancer. Pharmacol Ther 161: 79-96, 2016

10. Song JL, Chen C, Yuan JP and Sun SR: Progress in the clinical detection of heterogeneity in breast cancer. Cancer Med 5: 3475-3488, 2016.

11. Shen H and Li Z: miRNAs in NMDA receptor-dependent synaptic plasticity and psychiatric disorders. Clin Sci (Lond) 130: 1137-1146, 2016

12. Su W, Aloi MS and Garden GA: MicroRNAs mediating CNS inflammation: Small regulators with powerful potential. Brain Behav Immun 52: 1-8, 2016.

13. Zhou W, Zou B, Liu L, Cui K, Gao J, Yuan S and Cong N: MicroRNA-98 acts as a tumor suppressor in hepatocellular carcinoma via targeting SALL4. Oncotarget 7: 74059-74073, 2016.

14. Yang L, Liang H, Wang Y, Gao S, Yin K, Liu Z, Zheng X, Lv Y, Wang L, Zhang CY, et al: MiRNA-203 suppresses tumor cell proliferation, migration and invasion by targeting Slug in gastric cancer. Protein Cell 7: 383-387, 2016.

15. Gao Y, Feng B, Han S, Lu L, Chen Y, Chu X, Wang R and Chen L: MicroRNA-129 in human cancers: From tumorigenesis to clinical treatment. Cell Physiol Biochem 39: 2186-2202, 2016.

16. Ren W, Li C, Duan W, Du S, Yang F, Zhou J and Xing J: MicroRNA-613 represses prostate cancer cell proliferation and invasion through targeting Frizzled7. Biochem Biophys Res Commun 469: 633-638, 2016.

17. Hao W, Luo W, Bai M, Li J, Bai X, Guo J, Wu J and Wang M MicroRNA-206 inhibited the progression of glioblastoma through BCL-2. J Mol Neurosci 60: 531-538, 2016.

18. Liang AL, Zhang TT, Zhou N, Wu CY, Lin MH and Liu YJ MiRNA-10b sponge: An anti-breast cancer study in vitro. Oncol Rep 35: 1950-1958, 2016.

19. Du S, Li H, Sun X, Li D, Yang Y, Tao Z, Li Q and Liu K: MicroRNA-124 inhibits cell proliferation and migration by regulating SNAI2 in breast cancer. Oncol Rep 36: 3259-3266, 2016.

20. Wang L, Sun H, Wang X, Hou N, Zhao L, Tong D, He K, Yang Y, Song T, Yang J and Huang C: EGR1 mediates miR-203a suppress the hepatocellular carcinoma cells progression by targeting HOXD3 through EGFR signaling pathway. Oncotarget 7 : 45302-45316, 2016

21. Gomes BC, Martins M, Lopes P, Morujão I, Oliveira M, Araújo A Rueff J and Rodrigues AS: Prognostic value of microRNA-203a expression in breast cancer. Oncol Rep 36: 1748-1756, 2016.

22. Wu H and Zhang J: Decreased expression of TFAP2B in endometrial cancer predicts poor prognosis: A study based on TCGA data. Gynecol Oncol 149: 592-597, 2018.

23. Nasif D, Campoy E, Laurito S, Branham R, Urrutia G, Roqué M and Branham MT: Epigenetic regulation of ID4 in breast cancer: Tumor suppressor or oncogene? Clin Epigenetics 10: 111, 2018.
24. Zhang J, Lan Q and Lin J: Identification of key gene modules for human osteosarcoma by co-expression analysis. World J Surg Oncol 16: 89, 2018.

25. Wang YW, Zhang W and Ma R: Bioinformatic identification of chemoresistance-associated microRNAs in breast cancer based on microarray data. Oncol Rep 39: 1003-1010, 2018.

26. Hui HX, Hu ZW, Jiang C, Wu J, Gao Y and Wang XW: ZNF418 overexpression protects against gastric carcinoma and prompts a good prognosis. Onco Targets Ther 11: 2763-2770, 2018.

27. Wu N, Yan J, Han T, Zou J and Shen W: Integrated assessment of differentially expressed plasma microRNAs in subtypes of nonsyndromic orofacial clefts. Medicine (Baltimore) 97: e11224, 2018.

28. Yan L, Zhan $\mathrm{C}$, Wu J and Wang S: Expression profile analysis of head and neck squamous cell carcinomas using data from the cancer genome atlas. Mol Med Rep 13: 4259-4265, 2016.

29. Hou L, Lin Z, Ni Y, Wu Y, Chen D, Song L, Huang X, Hu H and Yang D: Microarray expression profiling and gene ontology analysis of long non-coding RNAs in spontaneously hypertensive rats and their potential roles in the pathogenesis of hypertension. Mol Med Rep 13: 295-300, 2016.

30. Li GM, Zhang CL, Rui RP, Sun B and Guo W: Bioinformatics analysis of common differential genes of coronary artery disease and ischemic cardiomyopathy. Eur Rev Med Pharmacol Sci 22: 3553-3569, 2018

31. Zhou W, Ma CX, Xing YZ and Yan ZY: Identification of candidate target genes of pituitary adenomas based on the DNA microarray. Mol Med Rep 13: 2182-2186, 2016.

32. Xu F, Gao F, Liu Y, Wang Z, Zhuang X, Qu Z, Ma H, Liu Y, Fu C, Zhang $\mathrm{Q}$ and Duan X: Bioinformatics analysis of molecular mechanisms involved in intervertebral disc degeneration induced by TNF- $\alpha$ and IL-1 $\beta$. Mol Med Rep 13: 2925-2931, 2016.

33. Huo W, Du M, Pan X, Zhu X, Gao Y and Li Z: miR-203a-3p.1 targets IL-24 to modulate hepatocellular carcinoma cell growth and metastasis. FEBS Open Bio 7: 1085-1091, 2017.

34. Kara M, Yumrutas O, Ozcan O, Celik OI, Bozgeyik E, Bozgeyik I and Tasdemir S: Differential expressions of cancer-associated genes and their regulatory miRNAs in colorectal carcinoma. Gene 567: 81-86, 2015.

35. Liu W, Dong Z, Liang J, Guo X, Guo Y, Shen S, Kuang G and Guo W: Downregulation of potential tumor suppressor mir-203a by promoter methylation contributes to the invasiveness of gastric cardia adenocarcinoma. Cancer Invest 34: 506-516, 2016.

36. Riemann A, Reime S and Thews O: Hypoxia-related tumor acidosis affects MicroRNA expression pattern in prostate and breast tumor cells. Adv Exp Med Biol 977: 119-124, 2017.

37. Lin QH, Zhang KD, Duan HX, Liu MX, Wei WL and Cao Y: ERGIC3, which is regulated by miR-203a, is a potential biomarker for non-small cell lung cancer. Cancer Sci 106: 1463-1473, 2015.

38. Liu Y, Dong Z, Liang J, Guo Y, Guo X, Shen S, Kuang G and Guo W: Methylation-mediated repression of potential tumor suppressor miR-203a and miR-203b contributes to esophageal squamous cell carcinoma development. Tumour Biol 37: 5621-5632, 2016.

39. Costa-Silva DR, Barros-Oliveira MD, Borges RS, Tavares CB, Borges US, Alves-Ribeiro FA, Silva VC and Silva BB: Insulin-like growth factor 1 gene polymorphism and breast cancer risk. An Acad Bras Cienc 88: 2349-2356, 2016.

40. De Santi M, Annibalini G, Barbieri E, Villarini A, Vallorani L, Contarelli S, Berrino F, Stocchi V and Brandi G: Human IGF1 pro-forms induce breast cancer cell proliferation via the IGF1 receptor. Cell Oncol (Dordr) 39: 149-159, 2016.

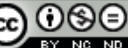

This work is licensed under a Creative Commons Attribution-NonCommercial-NoDerivatives 4.0 International (CC BY-NC-ND 4.0) License. 